\title{
3. Erste Schritte zur modernisierten Fürsorge: Teilreformen und Sondergesetze im Vorfeld umfassender Reform
}

Trotz der vom Bundesinnenministerium bis Herbst 1955 betriebenen Hinauszögerung einer umfassenden Reform wurde das Fürsorgerecht bereits in dieser Zeit in wesentlichen Teilbereichen erheblich verrechtlicht (Rechtsanspruch auf Fürsorge, starke Beschränkung der Kostenersatzpflicht, klar fixierte Mehrbedarfsmerkmale) und standardisiert (Warenkorb, einheitliche und weitgehend von sozialer Gruppenzugehörigkeit unabhängige Mehrbedarfe) und übernahm damit wesentliche Elemente sozialpolitischer Modernisierung.

\section{Die Einschränkung der Rückerstattungspflicht 1951}

Laut $\int \mathbb{S} 25$, 25a RFV hatte der Unterstützte - gegebenenfalls sein Ehegatte, seine Eltern, Kinder oder Erben - dem Fürsorgeverband die aufgewendeten Kosten bis auf gewisse Ausnahmen zu ersetzen; er war jedoch „berechtigt, den Ersatz zu verweigern, soweit und solange er kein hinreichendes Vermögen oder Einkommen“ hatte ${ }^{344}$. Richtlinien der Reichsregierung hatten 1934 verschiedene Härtefälle ausgenommen, eine sechsmonatige Schonfrist eingeführt und eine Grenze des wiedererlangten Arbeitseinkommens definiert (dreifacher Richtsatz), bis zu der kein Ersatz gefordert werden durfte. ${ }^{345}$ Diese Richtlinien galten auch nach Kriegsende weiter, wurden aber in den Ländern unterschiedlich verändert und ergänzt. Aus seuchenhygienischen Gründen war 1942 außerdem die Tuberkulosehilfe von der Rückerstattungspflicht ausgenommen worden.

Angesichts der offensichtlichen Schwierigkeiten, wieder zu „hinreichendem Einkommen und Vermögen zu gelangen“, hatte $\ 25$ RFV jetzt allerdings eher deklamatorische als praktische, allenfalls eine gewisse abschreckende Wirkung. Allerdings trug die unpopuläre Rückerstattungspflicht zum schlechten Image der öffentlichen Fürsorge bei, setzte sie die Hilfe doch gleichsam unter einen moralischen Vorbehalt und war dazu angetan, den Unterstützten verglichen mit dem Empfänger anderer Sozialleistungen zu degradieren. Anders als der „klassische“ Bezieher von öffentlicher Fürsorge galten jedoch gerade Vertriebene, die Familien von Kriegsgefangenen oder Ausgebombte als unverschuldet in Not geraten, und schienen Erstattungsforderungen an diese Gruppen daher nicht mehr legitim. Auf

${ }^{344}$ RFV in der Fassung der Zweiten Notverordnung vom 5.6.1931, geändert durch Reichsgesetz vom 22.12.1936 und die Dritte Vereinfachungsverordnung vom 11.5.1943. Nicht zu erstatten waren die Kosten der Wochenfürsorge, der Erwerbsbefähigung Blinder, Taubstummer und Körperbehinderter, der Fürsorge für Minderjährige sowie der Krankenhilfe im Rahmen der Seuchenbekämpfung; die Ersatzpflicht erlosch nach vier Jahren, vgl. $\iint$ 25-25d RFV in der genannten Fassung.

345 Runderlaß des Reichsarbeitsministers und Runderlaß des Preußischen Ministers des Innern über Rückforderungen der Kosten der öffentlichen Fürsorge vom 10.11.1934, RABl. I, S. 265; vgl. dazu NDV 28 (1948), S. 200ff. Danach war Kostenersatz in der Regel nicht $\mathrm{zu}$ verlangen von Unterstützten mit mindestens drei unterhaltsberechtigten Kindern, von über Sechzigjährigen sowie von denjenigen, die Pflichtarbeit geleistet hatten. Zu weiteren Ausnahmen in den 1930er Jahren vgl. Willing, Vorgeschichte, S. 594f. 
Anweisung der britischen Militärregierung wurden in der britischen Zone im Laufe des Jahres 1947 zunächst die Kriegsheimkehrer von der Ersatzpflicht für Leistungen an ihre Angehörigen befreit. ${ }^{346}$ Im Juni 1948 nahm Nordrhein-Westfalen nach den NS-Verfolgten auch die Vertriebenen von der Verpflichtung aus, Schleswig-Holstein 1949 die Kriegsopfer. ${ }^{347}$ In den Ländern der französischen Zone und in Groß-Berlin bestanden z.T. ähnliche Regelungen.

Anders in der US-Zone: Der Logik einer konsequenten Einheitsfürsorge gemäß, lehnte das Regional Government Coordinating Office im Mai 1947 entsprechende Pläne des Länderrats ab. Vielmehr solle die Rückerstattungspflicht „eher von der Finanzkraft des früheren Fürsorgeempfängers als von der Ursache seines Notstandes oder der sozialen Schicht, zu der er gehört, abhängig gemacht werden“348. Dies lag ganz auf der Linie Polligkeits, der Anfang 1948 einen Verordnungs-Entwurf zur Erstattungsfrage dem Wohlfahrtsausschuß des Länderrats zuleitete. ${ }^{349}$ Anlaß war die Forderung des hessischen Landtags nach einer Sistierung der Rückerstattungspflicht. ${ }^{350}$ Polligkeit wollte mit seinem Entwurf dem zunehmenden Druck von Länder- und Gemeindeparlamenten begegnen und ging, wie er selbst meinte, „bis an die Grenze des Vertretbaren“, wenn er bei sämtlichen Kriegsbetroffenen nur noch in Ausnahmefällen die Rückerstattung vorsah; der Entwurf habe aber gegenüber deren völliger Beseitigung „den Vorzug, dass er den Grundsatz der Subsidiarität mit seiner Tendenz der Erziehung zur Selbstverantwortung bestehen läßt“351. Während DST und DLT aber allenfalls (unverbindlichere) Richtlinien konzedieren wollten, plädierte Polligkeit für die Form der schneller wirksamen und im Interesse der Kriegsfolgenhilfe-Modalitäten stärker vereinheitlichenden Rechtsverordnung.

Nach langen Auseinandersetzungen kamen die Ländervertreter im Wohlfahrtsausschuß schließlich den kommunalen Bedenken weit entgegen, und am 16. September 1948 empfahl das Direktorium des Länderrats einen abgemilderten Musterentwurf nur mehr für Richtlinien den Ländern zur Einführung. ${ }^{352}$ Der Entwurf ging von der prinzipiellen Gleichbehandlung aller Unterstützten aus und basierte im übrigen auf den Richtlinien von 1934. Neu eingeführt wurde neben einer Klausel zum Schutz auch kleinerer Vermögen eine (angesichts der amerikanischen Vorgaben) bewußt allgemein gehaltene Regelung, wonach der Fürsorgeverband „bei den Personen, welche früheres Einkommen oder Vermö-

346 Vgl. NDV 28 (1948), S. $99 f$.

347 Vgl. insgesamt ebenda, S. 200ff.; NDV 30 (1950), S. 41ff.; 31 (1951), S.222; Krug von Nidda, Polligkeit, S. 258f.

348 Vgl. Charles D. Winning, Regional Government Coordinating Office, an den Generalsekretär des Länderrates Erich Roßmann am 19.5.1947, Übers. in: LAB, B Rep. 142-9, 1291; auszugsweise in: NDV 30 (1950), S. 41.

349 Vgl. „Entwurf einer Verordnung über die Begrenzung der Pflicht zum Ersatz von Fürsorgekosten" vom 26.1.1948, LAB, B Rep. 142-9, 1291.

350 Am 31.1.1948 setzte der hessische Arbeitsminister die Rückerstattungspflicht in einer Dienstanweisung bis auf weiteres aus, ebenda.

351 Polligkeit an DST, 23.2.1948, ebenda.

352 Vgl. „Musterentwurf für Richtlinien über die Rückführung von Fürsorgekosten“ vom 16. 9. 1948, ebenda; teilweise abgedruckt in: NDV 28 (1948), S. $201 \mathrm{f}$. 
gen unverschuldet verloren haben, die Notwendigkeit und Möglichkeit der Wiederbeschaffung der verlorenen Lebensgrundlagen zu berücksichtigen “ habe (Art. II Nr.5). Diese Vorgabe sollte den Vertriebenen und allen anderen, die durch den Krieg und seine Folgen Einkommen oder Vermögen verloren hatten, zugute kommen. Der Musterentwurf wurde 1948/49 von Bayern und Hessen sowie innerhalb der französischen Zone modifiziert von Württemberg-Hohenzollern und Baden übernommen, ansonsten bestanden die unterschiedlichen Länderregelungen fort.

Anfang Februar 1950 forderte der Bundestag mit den Stimmen aller Fraktionen die Bundesregierung auf, einen Gesetzentwurf zur Rückerstattungspflicht vorzulegen: Politisch, rassisch und religiös Verfolgte, ehemalige Kriegsgefangene, Vertriebene und Bombengeschädigte sowie deren Familien sollten jetzt bundesweit einheitlich von der Pflicht befreit werden. ${ }^{353}$ Bis zum Erlaß des Gesetzes sollten die Länder darum ersucht werden, für diese Gruppen die Rückzahlung auszusetzen. Die Berichterstatterin des Fürsorgeausschusses, Maria Niggemeyer (CDU), erklärte zur Begründung, die „soziale Notlage“ dieses Personenkreises sei „nicht selbstverschuldet, sondern herbeigeführt durch ein System, an dessen Folgen wir alle noch tragen - das nationalsozialistische System -, durch die Katastrophe des verlorenen Krieges, dessen Lasten wir ja auch alle zu tragen haben“. Der Antrag ziele daher auf eine Änderung des $\mathbb{S} 25 \mathrm{RFV}$, denn der Ausschuß könne es „nicht etwa dem Ermessen des Leiters eines Wohlfahrtsamtes oder Landesbezirksfürsorgeamtes überlassen [...], hier von sich aus zu entscheiden, ob im Einzelfall auf die Rückzahlung verzichtet werden soll“.354

Doch obwohl, wie die Antragstellerin der FDP zu Recht anmerkte, ein solches Gesetz keine neuen Geldmittel erfordern würde ${ }^{355}$, waren die Reaktionen der Fürsorgeträger und ihrer Verbände auf den Vorstoß des Bundestages geteilt: Fürsorgepraktiker aus Städten des gesamten Bundesgebietes erhoben kaum gewichtige Einwände, denn weder die genannten Gruppen noch andere Unterstützte seien „jetzt abgesehen von verschwindenden Ausnahmen, in der Lage, einen Pfennig zu erstatten“ 356 , oder müßten dies aufgrund besonderer Ländergesetze schon jetzt nicht mehr. Die Verpflichtung habe „für die praktische Arbeit [...] überwiegend moralische und psychologische Bedeutung “357; während der Kölner Beigeordnete Busch befürchtete, sie verschrecke allenfalls „die ordentlichen verschämten Armen, denen man an sich gerne helfen möchte" 358 , wandten sich andere wie der Bremer Senator van Heukelum gegen eine völlige Abschaffung der Ersatzpflicht,

353 Vgl. Sitzung des Bundestags am 1.2.1950, BT, 1. Wp. 1949, Sten. Ber., Bd.2, S. $1038 \mathrm{ff}$. Der Wortlaut des Antrags sah nur eine einheitliche Regelung der Rückerstattungspflicht vor, doch der zugrundeliegende Bericht des Ausschusses für Fragen der öffentlichen Fürsorge sowie die Debattenredner wünschten eindeutig deren Abschaffung für die genannten Personenkreise.

354 Abg. Niggemeyer (CDU) am 1.2.1950, ebenda, S. $1038 f$.

355 Vgl. Abg. Ilk (FDP), ebenda, S. 1043.

356 So der Trierer Oberbürgermeister Raskin, an den Städteverband Rheinland-Pfalz, 3. 3. 1950, LAB, B Rep. 142-9, 1291.

357 Schreiben des Wohlfahrtsamtes der Stadt Esslingen an den DST, 28. 2. 1950, ebenda.

358 Busch an Muthesius, 15.3.1950, ebenda. 
da durch ihre abschreckende Wirkung der „Wille zur Selbsthilfe [...] gestärkt“ werde ${ }^{359}$. Die kommunalen Spitzenverbände Bayerns, die auch in anderem $\mathrm{Zu}$ sammenhang gern als glühende Verteidiger fürsorgebehördlicher Ermessensfreiheiten auftraten, sahen gar das „Grundgefüge der zur Deckung des notwendigen Lebensunterhaltes bestimmten Sozialleistungen [...] erschüttert“, käme es zu dieser „Durchbrechung elementarster Grundsätze des Fürsorgerechts“, und rechneten mit erheblichen finanziellen Einbußen. ${ }^{360}$ Der stellvertretende DV-Vorsitzende Kurt Blaum wie der stellvertretende Hauptgeschäftsführer des DLT Schlüter wandten sich vor allem gegen eine Einschränkung der Erstattungspflicht per Gesetz, da dieses nicht den jeweiligen Länderbesonderheiten Rechnung tragen könne und nur schwerfällig an sozialpolitische Veränderungen anzupassen sei; außerdem lehre „die Vergangenheit, daß gesetzliche Regelungen Wünsche anderer Kreise wecken, deren Erfüllung sozial- und finanzpolitisch nachher nicht möglich ist". ${ }^{361}$ Sie befürworteten daher flexibel und individueller zu handhabende Verwaltungsrichtlinien auf der Grundlage des alten Musterentwurfs von 1948. Wie bei der zur gleichen Zeit akuten Frage der Anrechnung von Rentenleistungen sollte also alles getan werden, um die Begründung einer neuen Gruppenfürsorge $\mathrm{zu}$ verhindern. Auch Muthesius warnte vor den unübersehbaren Auswirkungen für die Fürsorgereform, falls der Bundestag möglicherweise noch weitere Gruppen in ein Sondergesetz einbeziehen würde. Er konstatierte allerdings, daß die Entwicklung „auf eine immer stärkere Zurückdrängung“ der Ersatzpflicht hinauslaufe, so daß deren künftige Rolle in einem neuen Bundesfürsorgegesetz ohnehin gründlich zu überdenken sei; daher rate er dringend davon ab, ,aus einem $\mathrm{Zu}$ fallsanlaß heraus eine isolierte Novellierung des $₫ 25 \mathrm{RFV}$ ins Werk zu setzen“..362 Um ein Präzedenz-Gesetz zu umgehen und um dem Bundestag das Heft aus der Hand zu nehmen, plädierte der Fachjurist dafür, die Ermächtigung des $\ 25$ RFV zu nutzen, wonach der Bundesinnenminister (mit dem Bundesarbeitsminister) per Rechtsverordnung Einkommens- und Vermögensgrenzen für eine Befreiung von der Rückerstattung bestimmen konnte. In Anlehnung an den Musterentwurf der amerikanischen Zone könne in dieser Verordnung durchaus den Bundestagswünschen entsprochen werden.

Diese „kleine Lösung“ entsprach der Linie der Sozialabteilung, und so teilte der Bundesinnenminister dem Bundestag am 23. Mai 1950 mit, daß angesichts der nicht unbeachtlichen Einwände der meisten Länder, der kommunalen Spitzenverbände und des DV nun nach einem Weg gesucht werde, „der einerseits dem berechtigten Wunsche nach einem Schutz gegen unsoziale Erstattungsansprüche der Fürsorgeträger Rechnung trägt, der andererseits aber auch die aufrecht zu erhaltenden Grundsätze des deutschen Fürsorgerechts berücksichtigt" ${ }^{\text {". }}$.63

\footnotetext{
359 G. van Heukelum an BMI am 7.3.1950, Abschrift, ebenda.

360 Gemeinsames Schreiben des Bayerischen Städteverbandes und des Landkreisverbandes Bayern an das Bayerische Staatsministerium des Innern, 13.1.1950, ebenda.

361 Schlüter (DLT) an BMI, 9.3.1950; vgl. auch Blaum an BMI, 1.3.1950, ebenda.

362 Muthesius an BMI, 14.3.1950, ebenda.

363 Antwort des BMI auf FDP-Anfrage Nr. 72 vom 23.5.1950, BT, 1. Wp. 1949, Anlagen, Bd. 4, Drs. 987.
} 
Knapp zwei Wochen zuvor hatte das Innenministerium bereits einen solchen Verordnungsentwurf an DST, DLT, DV sowie jeweils ein Länderministerium der drei ehemaligen Besatzungszonen gesandt. ${ }^{364}$ Der Entwurf sah wie die Musterrichtlinien von 1948 im Sinne der Einheitsfürsorge besondere Schonung nicht nur für die vom Bundestag genannten Gruppen vor, sondern für jeden, der „früheres Einkommen oder Vermögen aus politischen, rassischen oder religiösen Gründen oder durch den Krieg bzw. die Kriegsfolgen verloren hat (z.B. Heimkehrer, Kriegssachgeschädigte, Währungsgeschädigte)“ (Art. I Ziff. 7). Auf einer Besprechung Ende Mai einigten sich die Beteiligten jedoch auf einen formal besser ausgearbeiteten, in der Sache rigideren Gegenentwurf des DV. ${ }^{365}$ Dieser bildete die Grundlage für den schließlich im Spätsommer vorgelegten endgültigen Entwurf. Danach war bei der großen Gruppe der Kriegsbetroffenen, also auch den Kriegsbeschädigten, Evakuierten oder DDR-Flüchtlingen, „von der Geltendmachung von Ersatzansprüchen abzusehen, wenn und solange die Wiederherstellung einer normalen Lebensgrundlage durch die Heranziehung zum Kostenersatz beeinträchtigt würde“.366 Getreu dem Individualprinzip sollte die Entscheidung darüber, was als „normale Lebensgrundlage“ zu gelten habe, also doch beim örtlichen Fürsorgebeamten bleiben, dem allerdings für die betroffenen Personengruppen schonendes Vorgehen verordnet wurde. Die praktische Bedeutung der Ersatzpflicht spielte die Begründung zum Entwurf herunter: Da „eine angemessene Zeitspanne“ erforderlich sei, um auf gesicherter Lebensgrundlage wieder zu Einkommen und Vermögen zu gelangen, der Ersatzanspruch gem. $\int 25 \mathrm{~b}$ RFV aber nach vier Jahren erlösche, werde dieser ohnehin weitgehend hinfällig werden. ${ }^{367}$

Nichtsdestoweniger müsse die Rückerstattung bestehen bleiben, um der vorrangigen Verpflichtung zur Selbsthilfe „die gesetzliche Ausdrucksform“ zu erhalten, zumal die langfristigen Folgen einer völligen Abschaffung „sowohl in sozialpädagogischer wie auch in finanzieller Hinsicht“ nicht abzusehen seien. ${ }^{368}$ Diese Argumentation überzeugte auch den Bundestagsausschuß für Fragen der öffentlichen Fürsorge, der den Entwurf am 29. September 1950 einstimmig billigte und nicht mehr auf einer gesetzlichen Regelung beharrte. ${ }^{369}$

Die Zustimmung der Länder war jetzt umso wahrscheinlicher, als am 28. November 1950 das Erste Überleitungsgesetz in Kraft trat, mit dem der Bund den Großteil der Fürsorgekosten für die von der Neuregelung profitierenden Kriegsfolgenhilfeempfänger selbst übernahm. Jetzt begünstigte der Bundesrat sogar selbst die Fürsorgeempfänger, indem er bestimmte, daß für Leistungen an die Familien von Kriegsgefangenen und Vermißten kein Ersatz verlangt werden durfte,

364 Vgl. BMI an DST etc. am 10.5.1950 nebst Anlage „Vorläufiger Entwurf einer Verordnung über den Ersatz von Fürsorgekosten“, LAB, B Rep. 142-9, 1291.

365 Vgl. Aktenvermerk Spahn (DST) betr. Besprechung am 26.5.1950 im BMI, nebst Gegenentwurf, ebenda.

366 BR 1950, Anlagen, Drs. 964/50.

367 Vgl. Begründung zum Regierungsentwurf, S. 4, ebenda.

368 Ebenda, S. 2.

369 Vgl. BMI Lehr an den Präsidenten des Bundesrates, 16.11.1950, BR 1950, Anlagen, Drs. $964 / 50$. 
so die Regelung der ehemaligen britischen Zone auf das Bundesgebiet ausdehnte und der Bundestagsforderung zumindest für einen Personenkreis wirklich Rechnung trug. ${ }^{370}$ Mit dieser Ergänzung wurde die „Verordnung über den Ersatz von Fürsorgekosten“ am 30. Januar 1951 erlassen und trat am 3. März 1951 in Kraft. ${ }^{371}$

Wie weit jedoch gerade in einem dem Individualprinzip verpflichteten System rechtliche Theorie und exekutive Praxis auseinander liegen können, belegten wenig später zwei Rundschreiben des Bundesinnenministeriums: Ende April bat der Bundesinnenminister die zuständigen Länderminister, dafür zu sorgen, daß die neue Verordnung auch wirklich angewendet werde und von seiten der Fürsorgebeamten „dabei den Unterstützten oder Ersatzpflichtigen dasjenige Maß an menschlichem und sozialem Verständnis entgegengebracht wird, auf das sie berechtigten Anspruch erheben dürfen“372. Gleichzeitig wandte er sich gegen Ersatzforderungen an aus der Kriegsgefangenschaft heimkehrende Väter unehelicher Kinder, gesetzlich unterhaltspflichtige ehemalige NS-Verfolgte, Flüchtlinge etc. und stellte klar, daß das über der Grenze liegende Einkommen keineswegs automatisch voll zum Ersatz herangezogen werden dürfe. Wie wenig jedoch die der Verordnung zugrundeliegende Idee, auch bei der Rückerstattung - und damit zugunsten des Hilfeempfängers - die individuelle Lage zu berücksichtigen, an der behördlichen Basis Eingang gefunden hatte, wurde mit Inkrafttreten des Gesetzes zu Art.131 GG vom 11. Mai 1951 deutlich: Als bislang von der Fürsorge unterstützte ehemalige öffentliche Bedienstete wieder Versorgungsansprüche erhielten, forderten Fürsorgeverbände von diesen Kostenersatz. Das Bundesinnenministerium, das die Befriedung der „131er" nicht durch knauserige Fürsorgebehörden gefährdet sehen wollte, erklärte daraufhin im Juni dieses Vorgehen für regelwidrig und vermerkte mit drohendem Unterton, daß der "gesunde Grundsatz“ der Rückerstattungspflicht nur dann überhaupt noch aufrechterhalten werden könne, „wenn die Fürsorgeverbände bei der Frage der Geltendmachung von Ersatzansprüchen weniger rein finanzielle Gesichtspunkte, als allgemein soziale und menschliche Erwägungen [...] zugrunde legen“373.

Der radikale Schritt einer vollständigen Abschaffung der Rückerstattungspflicht war 1951 also nicht getan worden. Ein solcher Schritt hätte eine Annäherung an eine vorbehaltlose Garantie des Existenzminimums für jeden Staatsbürger, unabhängig von der Ursache seiner Notlage, bedeutet, die rechtliche Position jedes Unterstützten verbessert, die Hemmschwelle zur Inanspruchnahme der Hilfe herabgesetzt und möglicherweise die Mißbrauchsgefahr erhöht. Doch so weit war der Bundestag mit seiner Forderung gar nicht gegangen; vielmehr hielten auch die Bundestagsfraktionen explizit an der Unterscheidung von selbstverschuldeter (individueller) und unverschuldeter (Massen-)Not fest, wenn sie die Befreiung von der Ersatzpflicht nur für die Flüchtlinge usw. forderten. Auch der

370 Vgl. Sitzung des Bundesrates am 15.12.1950, BR 1950, Sten. Ber., S. $73 f$.

371 Vgl. BGBl. I S. 154.

372 RdSchr. d. BMI betr. Ersatz von Fürsorgekosten vom 30.4.1951, GMBl. S. 132, Zitat S. 133.

373 RdSchr. d. BMI betr. Ersatz von Fürsorgekosten vom 20.6.1951, GMBl. S. 169, Zitat ebenda. 
Wunsch, der öffentlichen Fürsorge Sanktionsmöglichkeiten gegenüber den (tatsächlich oder vermeintlich) selbstverschuldet Hilfsbedürftigen zu erhalten, dürfte dazu beigetragen haben, daß sich der durch den Fürsorgeausschuß vertretene Bundestag mit einer Rechtsverordnung anstelle eines Gesetzes zufriedengab. Die traditionelle Scheidung von "würdigen" und "unwürdigen“ Armen war damit auch Anfang der fünfziger Jahre noch lebendig und stand in enger Korrelation zur politischen Bedeutung und organisatorischen Durchschlagkraft der jeweiligen Armutsgruppen.

Nichtsdestoweniger bewirkte die Verordnung von 1951 in der Praxis eine Aufweichung der Rückerstattungspflicht, die im Fürsorgeänderungsgesetz von 1953 weitergeführt wurde. Und im Vorfeld der Bundestagswahlen 1953 setzte der Bundestag für die größte (und politisch wichtigste) Gruppe der Kriegsbetroffenen schließlich doch noch seine Forderung vom Februar 1950 in anderem Rahmen um: $\mathbb{9} 91$ des Bundesvertriebenengesetzes vom 19. Mai 1953 befreite de facto Heimatvertriebene (und DDR-Flüchtlinge) nun eindeutig von der Ersatzpflicht, $\mathbb{1} 19$ des Bundesevakuiertengesetzes vom 14.Juli 1953 (\$ 19) die Evakuierten. Die finanzielle Bedeutung der Erstattungspflicht nahm so immer weiter ab, zumal der behördliche Arbeitsaufwand in keinem Verhältnis mehr zum finanziellen Ertrag stand. ${ }^{374}$ Bei den Vorarbeiten zum BSHG sollte die Frage - wie Muthesius vorhergesagt hatte - wieder auf der Tagesordnung stehen.

\section{Sicherungssystematische „Flurbereinigung “ durch das Mebrbedarfs-Modell: das Fürsorgeänderungsgesetz von 1953}

Die Frage der sogenannten Freilassung von Teilen anderer Sozialleistungseinkommen war seit Einführung von RFV und RGr. immer wieder Anlaß für Auseinandersetzungen zwischen kommunalen Trägern und DV auf der einen, Rentnerund Kriegsopferorganisationen, Gewerkschaften und der sie unterstützenden Parteien auf der anderen Seite. Bei diesen oft mit dem schweren Geschütz der theoretischen Systematik sozialer Sicherung geführten Diskussionen ging es darum, ob die Erhöhung einer Rente bei der Berechnung der zusätzlichen Fürsorgeunterstützung voll in Ansatz zu bringen sei; ob also die Erhöhung tatsächlich dem betreffenden Rentenempfänger oder aber dem Fürsorgeverband zugute kommen würde, der nun weniger oder gar keine Unterstützung mehr zahlen mußte.

Bereits 1925 waren die Sozialbürokratien der Länder und Gemeinden gegen ein Initiativgesetz des Reichstages Sturm gelaufen, das eine feste Freilassung von Rententeilen bei der Fürsorgebemessung vorsah. ${ }^{375}$ Daraufhin kassierte die Reichsregierung das Gesetz, konzedierte dem Reichstag und den Rentner- und Kriegsopferverbänden allerdings die bereits erwähnte Verordnung vom 7. September 1925,

374 Der Bochumer Stadtdirektor klagte bereits im Sommer 1951, daß durch die neue Verordnung ursprünglich per Landesrecht von der Ersatzpflicht ganz Befreite (Flüchtlinge, Heimkehrer etc.) nun wieder auf ihre Erstattungsfähigkeit hin überprüft werden müßten, was erhebliche Mehrarbeit bringe, während die Nachprüfungen in 99\% der Fälle negativ ausfielen; vgl. Schreiben an den DST, 23.7.1951, LAB, B Rep. 142-9, 1291.

375 Vgl., auch zum Folgenden, Sachße/Tennstedt, Geschichte, Bd. 2, S. $179 \mathrm{ff}$. 
die die Aufstellung von Richtsätzen mit besonderen Zuschlägen (in der Regel mindestens ein Viertel über dem allgemeinen Richtsatz) für die Bezieher gehobener Fürsorge vorsah und insofern der fürsorgerischen Begünstigung bestimmter sozialer Gruppen Rechnung trug. In der Zeit des Nationalsozialismus wurden in einem Durchführungserlaß zum „Gesetz über die Verbesserung der Leistungen in der Rentenversicherung"vom 24. Juli 1941 einheitliche Freilassungsbeträge festgesetzt. ${ }^{376}$ Darüber hinaus legten das Gesetz über Kleinrentnerhilfe vom 5.Juli 1934 und der Richtsatzerlaß vom Oktober 1941 wieder feste Mindestzuschläge für die Richtsätze der gehobenen Fürsorge fest, die infolge der Weltwirtschaftskrise weggefallen waren: Der Richtsatz für Sozialrentner etc. sollte um 15\%, der für Kriegsopfer und bestimmte Kleinrentner um 25\% höher als der allgemeine Richtsatz sein. ${ }^{377}$ Rentenbezieher waren jetzt also in doppelter Weise gegenüber anderen Hilfsbedürftigen bevorzugt.

Nach Ende des Krieges waren vielerorts zunächst leere Gemeindekassen und das alliierte Verbot der Gruppenfürsorge Anlaß, auch Renteneinkommen voll auf die Fürsorgeunterstützung anzurechnen, oder, wie es der NDV zustimmend formulierte, „das Subsidiaritätsprinzip zunächst wieder schärfer" durchzuführen. ${ }^{378}$ Die Besatzungsmächte legten allerdings auch hier den Grundsatz der Einheitsfürsorge unterschiedlich aus: Während in der britischen und der französischen Zone die Freilassungsbeträge von 1941 bestehenbleiben konnten, untersagten die Amerikaner deren Anwendung. Unterschiedlich angerechnet wurden auch die Rentenerhöhungen infolge des Sozialversicherungs-Anpassungsgesetzes ab Juni 1949, das die prekäre Lage der Sozialrentner durch Rentenzuschläge und die Einführung von Mindestrenten endlich verbessern sollte. $\mathrm{Zu}$ dieser Zeit unterstützten die Fürsorgeverbände des Vereinigten Wirtschaftsgebietes Sozialrentner mit schätzungsweise jährlich 50 Mio. DM zusätzlich und rechneten bei voller Anrechnung der Erhöhungen mit einer Entlastung von rund 20 Mio. DM pro Jahr. ${ }^{379}$ Doch die Ausschüsse für Arbeit des Wirtschafts- und des Länderrats empfahlen den Fürsorgeverbänden, die Rentenerhöhungen nicht oder nicht voll anzurechnen. ${ }^{380}$ Demgegenüber warnte der Sozialausschuß des DST vor einer erneuten „Doppelgleisigkeit der Sozialversicherung und der öffentlichen Fürsorge“ und empfahl, durch die volle Anrechnung erzielte Ersparnisse „gegebenenfalls für Neben- und Sonderleistungen der öffentlichen Fürsorge in besonderen Einzelfällen nach individueller Prüfung zu verwenden““. ${ }^{381}$ Doch auf entsprechende Landtagsbeschlüsse hin wurden wenig später in Niedersachsen und Rheinland-Pfalz die Freilassungs-

376 RGBl. I, S. 443. Nach dem „Runderlaß des Reichsarbeitsministeriums und des Reichsinnenministeriums über Behandlung der Leistungsverbesserungen der Rentenversicherung in der öffentlichen Fürsorge“ vom 28.7.1941, RMBliV., S. 1425, waren freizulassen: für hilfsbedürftige Versicherte 7 bzw. 6 RM, für Witwen/Witwer 5 RM, für Waisen 4 RM; vgl. NDV 29 (1949), S.255; ferner eine Auflistung der Freilassungsregelungen 1933-1945 in: NDV 30 (1950), S. 164.

377 Vgl. auch Sachße/Tennstedt, Geschichte, Bd. 3, S. $248 f$.

378 NDV 29 (1949), S. 252.

379 Vgl. ebenda, S. 143.

380 Vgl., auch zum Folgenden, ebenda, S. 255f.; NDV 30 (1950), S. 14.

381 Entschließung des DST-Sozialausschusses vom 2.7.1949, in: Jellinghaus, Betrachtungen zur Anwendbarkeit, S. 25f. 
beträge gegenüber 1941 teilweise mehr als verdoppelt, in Baden blieben die Rentenzuschläge gänzlich anrechnungsfrei. Alle anderen Länder beließen es entweder bei den Freilassungen von 1941 oder rechneten sämtliche Rentenerhöhungen voll an.

Obwohl dadurch gerade diejenigen benachteiligt wurden, deren Rente so klein war, daß sie durch die Fürsorge aufgestockt werden mußte, wurde im Nachrichtendienst des noch von Polligkeit geführten DV dieses Verfahren begrüßt: Die Erhöhung der Freilassungsbeträge führe praktisch zu einer neuen Gruppenfürsorge, wogegen „bei den bestehenden Notzeiten schwerste Bedenken geltend gemacht werden“ müßten, zumal nun auch andere Gruppen entsprechende Forderungen erheben könnten, so daß „die Höhe der Soziallasten die volkswirtschaftliche Kraft übersteige“382. Doch im neu konstituierten Bundestag stieß dieser Umgang mit den Rentenerhöhungen auf Kritik: Aus Anlaß eines Antrages der KPD-Fraktion vom 14. Oktober 1949 berieten die Ausschüsse für Sozialpolitik und für öffentliche Fürsorge gemeinsam, wie „die berechtigte Anschauung der Rentnerkreise berücksichtigt werden [könne], daß durch die Handhabung der Anrechnung der erhöhten Renten bei den Fürsorgeämtern eine Hand nehme, was die andere gegeben hätte“, und es zu einer „Serie von sehr großen sozialen Härten“383 gekommen sei. Vielen zusätzlich unterstützten Rentnern gehe es jetzt sogar schlechter als zuvor, da ihnen die Fürsorge die Sonderbeihilfen für Brennstoff etc. gestrichen habe. Der Berichterstatter Willy Fischer (SPD) brachte jetzt eine Freilassung in Form eines Prozentanteils des Richtsatzes ins Spiel, um auf diese Weise regionale und lokale Unterschiede besser berücksichtigen $\mathrm{zu}$ können ${ }^{384}$ - und den Fürsorgeträgern eine solche Freilassung schmackhafter zu machen. In mißverständlicher Formulierung beantragte der sozialpolitische Ausschuß daraufhin die Vorlage eines Gesetzentwurfes, „nach dem ein Hundertsatz der jeweiligen Fürsorgebeträge nicht angerechnet werden soll für die Empfänger von Leistungen aus der Sozialversicherung“385. Am 1. Februar nahm der Bundestag diesen Antrag einstimmig an. ${ }^{386}$ Einen Tag später fügte der Bundestag konsequent in den Gesetzentwurf zur Verbesserung von Leistungen an Kriegsopfer einen Passus ein, wonach die geplante Erhöhung der Kriegsopferrenten auf die Fürsorge nicht angerechnet werden dürfe, und fand dafür die Zustimmung des Bundesrates. ${ }^{387}$

Aus Sicht der Kostenträger der öffentlichen Fürsorge, zu denen in Kürze auch der Bund gehören sollte, bedeuteten diese Beschlüsse einen gefährlichen Dammbruch nun auch auf Bundesebene, dem bei jedem künftigen Renten- oder Versor-

382 NDV 29 (1949), S. 255, 162. Daß die Fürsorgeverbände allerdings nicht gleichsam naturgegeben jeder Freilassung widerstrebten, belegt eine Empfehlung des Arbeitsausschusses der westfälischen Fürsorgeverbände vom Sommer 1949, den Freilassungsbetrag auf $7 \mathrm{DM}$ zu erhöhen; vgl. ebenda, S. 162.

383 Abg. Willy Fischer (SPD) im Bundestag am 1.2.1950, BT, 1. Wp. 1949, Sten. Ber., Bd. 2, S. 1039.

384 Vgl. Fischer, ebenda, S. 1040.

385 Vgl. den Mündlichen Bericht des Ausschusses für Sozialpolitik vom 24.1.1950, BT, 1. Wp. 1949, Anlage, Bd.2, Drs. 453.

386 Vgl. BT, 1. Wp. 1949, Sten. Ber., Bd.2, S. 1043.

387 Vgl. Sitzung des Bundestages am 2.2.1950, ebenda, S.1062f., 1076ff.; Sitzung des Bundesrates am 16.2.1950, BR 1950, Sten. Ber., Bd.1, S. 241. 
gungsgesetz weitere folgen würden. Die Uneinheitlichkeit der Anrechnungsregelungen würde überdies die praktische Abwicklung der Kriegsfolgenhilfe deutlich erschweren. In enger Kooperation erarbeiteten die Sozialpolitische Abteilung des Bundesinnenministeriums, die zuständigen Abteilungen des Bundesfinanzministeriums, Ländervertreter, kommunale Spitzenverbände und der DV daher 1950/51 ein Konzept, das die Frage der Freilassungen und damit der „Gruppenfürsorge“ in einem Änderungsgesetz zu RFV und RGr. ein für allemal regeln sollte. ${ }^{388}$

Nachdem bereits unmittelbar nach den Bundestagsbeschlüssen der stellvertretende DV-Vorsitzende Blaum, kommunale Spitzenverbände und der von Polligkeit mobilisierte „Arbeitsstab Kriegsfolgenhilfe“ bei Innen- und Finanzministerium sowie im Finanzausschuß des Bundesrats schwerwiegende Bedenken gegen die Freilassungspraxis geltend gemacht hatten ${ }^{389}$, wurde auf einer Besprechung mit Vertretern der Länder, der Spitzenverbände der Kommunen und der freien Wohlfahrtspflege beschlossen, den „Arbeitsstab Polligkeit“ mit den Vorarbeiten für die Teilreform zu betrauen. Von dem Arbeitsstab erhoffte sich nicht zuletzt Finanzminister Schäffer eine angemessene Berücksichtigung der finanziellen Interessen von Ländern und Gemeinden wie des Bundes, der seit dem 1. April ja einen wesentlichen Teil der öffentlichen Fürsorgekosten zu tragen hatte. ${ }^{390}$

Im Juni 1950 meldete sich der „Reichsbund der Kriegs- und Zivilbeschädigten, Sozialrentner und Hinterbliebenen “ zu Wort und forderte die unbeschränkte Wiedereinführung der Gruppenfürsorge zugunsten der Rentner und Kriegsopfer. ${ }^{391}$ Zwar hoffte man im Innenministerium offensichtlich, durch das in Vorbereitung befindliche Bundesversorgungsgesetz solchen Forderungen den Wind aus den Segeln nehmen zu können, und spielte auf Zeit: Am 26. Juni erklärte das Ministerium in einem Zwischenbescheid an den Bundestag, die meisten Länder, die kommunalen Spitzenverbände, der DV und der Finanzausschuß des Bundesrates hätten sich gegen die Durchführung des Bundestagsbeschlusses vom Februar ausgesprochen; man suche daher nach einer für alle Fürsorgeempfänger gerechten Lösung, was zunächst eingehende Untersuchungen und Verhandlungen erfordere. ${ }^{392}$

Doch auch im Innenministerium ging man nicht davon aus, daß der Bundestag seinen einstimmigen Beschluß zurückziehen würde; vielmehr hielt Gottschick es für akzeptabel, alten und invaliden Hilfsbedürftigen wegen ihrer höheren Belastungen bestimmte, möglicherweise gestaffelte Beträge freizulassen. ${ }^{393}$ Anstelle sozialer Gruppenzugehörigkeit sollten also individuelle Tatbestände gesteigerter

\footnotetext{
388 Zum Folgenden vgl. Heisig, Armenpolitik, 1995, S. $61 \mathrm{ff}$.

389 Vgl. NDV 30 (1950), S. 54f.; Jellinghaus, Betrachtungen zur Anwendbarkeit, S. $21 \mathrm{ff}$.

390 Vgl. das bei Heisig, Armenpolitik, 1995, S. 64, ohne Datumsangabe zitierte Schreiben Schäffers an das BMI. Ein Grund dafür, daß der Arbeitsstab mit den Vorarbeiten betraut wurde, dürfte auch die Führungskrise innerhalb des sonst dafür prädestinierten DV gewesen sein.

391 Vgl. „Denkschrift zum Problem der Einheitsfürsorge“ des Reichsbundes, 12.6.1950, LAB, B Rep. 142-9, 1290.

392 Vgl. Antwort des BMI vom 26. 6. 1950, BT, 1. Wp. 1949, Anlagen, Bd. 5, Drs. 1115.

393 Vgl. Heisig, Armenpolitik, 1995, S. 65.
} 
Hilfsbedürftigkeit den Maßstab der Fürsorgebemessung bilden. Den Anlaß für solch konkrete Überlegungen gaben auch Entwicklungen auf einer ganz anderen Ebene: Gottschick rechnete damit, daß die Alliierten Hohen Kommissare im Rahmen der Lockerung des Besatzungsstatuts „in absehbarer Zeit“ die britischen und amerikanischen Verbote der gehobenen Fürsorge aufheben würden, so daß rechtzeitig von deutscher Seite aus entsprechende Maßnahmen vorzubereiten seien. ${ }^{394}$

Die quantitative Dimension des Problems war - wie so oft - eine Frage des Betrachters: Ende Juni 1950 wurden 136000 Sozialrentner in der öffentlichen Fürsorge laufend unterstützt; legte man die Zahl aller Sozialrentner zugrunde, so war ihr Anteil mit 3,1\% denkbar gering; ihr Anteil an allen Fürsorgeempfängern hingegen betrug 19,2\%, also immerhin ein Fünftel. ${ }^{395}$ Angesichts der Tatsache, daß die durchschnittliche Monatsrente einer Witwe in der Arbeiterrentenversicherung zu dieser Zeit 36,20 DM betrug ${ }^{396}$, der Richtsatz der öffentlichen Fürsorge im Bundesdurchschnitt für Alleinstehende bei 38,90 DM lag 397 , konnte also jede Änderung der Anrechnungsbestimmungen relativ schnell zumindest auf die Zahl der zu unterstützenden Rentenempfänger durchschlagen. Die Größenordnung der zweiten wichtigen Gruppe, der Kriegsopfer, war vor Inkrafttreten des BVG ähnlich: Am 30. Juni 1950 wurden 192000 Kriegsopfer von der öffentlichen Fürsorge laufend unterstützt, knapp 5\% aller ca. 4,3 Mio. Kriegsopfer, ihr Anteil in der Fürsorge hingegen belief sich auf 13,6\%.398

Auf eine offizielle Bitte von Kitz auf dem Fürsorgetag vom 6./7. Oktober 1950 hin begann der neue Fachausschuß I des DV mit den Vorarbeiten zur Lösung der Anrechnungsfrage. ${ }^{399}$ Die Verlagerung der Reformarbeiten von dem primär mit Finanzfragen befaßten, nunmehr ohnehin obsoleten Arbeitsstab in den Fürsorgefachverband DV war sicher von optischem Vorteil. In der Sache war allerdings für Kontinuität gesorgt, leitete doch der ehemalige stellvertretende Arbeitsstabs-Vorsitzende Muthesius den Fachausschuß, dem auch das ehemalige Arbeitsstabs-Mitglied Kitz angehörte.

Anfang Januar 1951 legten die Fürsorgereferenten der Länder zusammen mit den Vertretern des Innenministeriums, Muthesius sowie Elsholz und FischerMenshausen die weitere Marschroute fest: Da das Innenministerium im Falle der vollen Anrechnung anderer Sozialeinkommen erhebliche politische Schwierigkeiten erwartete, sollte die Freilassung künftig am Bedarf orientiert werden; bei den Richtsätzen sollte es bundesrechtliche Rahmenvorschriften geben, innerhalb derer die Länder zwar die Sätze frei gestalten könnten, für eine Überschreitung aber die

394 Vgl. Vermerk der DST-Geschäftsstelle vom 11.9.1950, LAB, B Rep. 142-9, 1291; NDV 30 (1950), S. 247, 255.

395 Angaben ohne West-Berlin. Vgl. Bundesministerium des Innern (Hg.), Fürsorge, S. 28. Über die (in dieser Frage letzthin entscheidenden) Kosten dieser Unterstützungen allerdings liegen keine repräsentativen Angaben vor.

396 Vgl. Frerich/Frey, Handbuch, Bd.3, S. 47, Tabelle 9 (Angabe für Anfang 1950, ohne Berlin-West).

397 Vgl. Bundesministerium des Innern (Hg.), Fürsorge, S. 16 (Angabe für 1949).

398 Berechnet nach: Statistisches Jahrbuch 1952, S. 354; vgl. auch Hudemann, Sozialpolitik, S. 517.

399 Vgl. Kitz, Aufgaben, S. 247; NDV 30 (1950), S. 265. 
Genehmigung des Bundes gemäß dem Ersten Überleitungsgesetz einholen müßten. ${ }^{400} \mathrm{Im}$ Schlepptau der Freilassungsfrage sollte also gleichzeitig ein weiteres wichtiges Anliegen geregelt werden: Beschlüsse einzelner Landtage über Richtsatzerhöhungen künftig stärker zu behindern und so nicht zuletzt den Bundeshaushalt vor unkalkulierbaren Kriegsfolgenhilfekosten zu schützen. Dem Bundestag war das u.a. dadurch plausibel zu machen, daß die Gruppenfürsorge ja auch und gerade auf unterschiedlichen Richtsätzen basiert hatte, ihre Abschaffung also auch eine Neuregelung der Richtsatzfrage erforderte. Wenn andererseits die Koppelung des Mehrbedarfs für Alte etc. an die Richtsätze im Bundestag durchgesetzt werden sollte, dann mußte allzu großer Freiheit bei deren Bemessung ein Riegel vorgeschoben werden. Letzteres widersprach natürlich den Interessen vieler kommunaler Träger, weshalb die kommunalen Spitzenverbände zunächst um ein verbrieftes Mitwirkungsrecht an deren Ausgestaltung bemüht waren und, als das nicht gelang, diese indirekt zu beeinflussen suchten.

Wie den berechtigten Wünschen der von Altersarmut Betroffenen und dem Bundestagsbeschluß Rechnung getragen werden sollte, ohne den eingeschlagenen Weg zur Einheitsfürsorge wieder zu verlassen, erläuterte Muthesius vor dem Fachausschuß I am 22. Februar 1951: „Es ist im Ergebnis dasselbe, ob wir einem Hilfsbedürftigen von der Einkommensseite her einen Teil seiner Rente nicht anrechnen oder ob wir ihm von der Bedarfsseite her einen höheren Bedarf zuerkennen, indem wir einen höheren Richtsatz anwenden. [...] Wir müssen versuchen, ausschließlich von der Bedarfsseite her eine Lösung zu finden ". ${ }^{401}$ Ganz so harmlos war die Sache natürlich nicht: Freilassungsbeträge, klar definiert und bundeseinheitlich, könnte der Bundestag bei seiner Renten- und Versorgungsgesetzgebung festlegen. Vom Richtsatz abhängige, also im Betrag unbestimmtere und regional unterschiedliche Mehrbedarfsbeträge hingegen könnten die zuständigen obersten Landesbehörden bzw. die von ihnen beauftragten Kommunen bestimmen. Wie Muthesius ein Jahr später vor dem Fürsorgeausschuß des Bundestages deutlich machte, hoffte er allerdings, daß gerade diese Orientierung am Bedarf letztlich zu einer klaren allgemeinen Definition eines Mindestlebensstandards führen müsse, dessen Sicherung er ja gerade dem fürsorgerischen Hilfsbedürftigkeits-Diktat entziehen wollte. 402

Am 23. Februar 1951 verabschiedete der DV-Vorstand eine vom Ausschuß vorbereitete entsprechende Entschließung und übersandte sie dem Innenministerium: Eine Lösung sei „vielleicht in der Weise möglich, daß allgemeine für alle Hilfsbe-

400 Vgl. Heisig, Armenpolitik, 1995, S. 65.

401 NDV 32 (1952), S.31. So hatte Muthesius bereits 1947 in seinem Kommentar zu den RGr. argumentiert, vgl. Muthesius, Grundlagen, S.91f., 94. Das von Muthesius befürwortete Verfahren wandte ein Teil der Fürsorgeverbände auch bisher schon an, wenn sie bei arbeitenden älteren oder erwerbsbeschränkten Unterstützten deren Einkommen nicht teilweise außer Ansatz ließen, wie in $\$ 8$ Abs. 5 RGr. vorgesehen, sondern stattdessen den Richtsatz überschritten, vgl. ebenda, S. 94; NDV 31 (1951), S. 89, oder indem sie bei besonderen Aufwendungen für Kinder, bei schwerer Krankheit u.a.m. Zuschläge zu den Richtsätzen vorsahen (so in Westfalen und Niedersachsen); vgl. NDV 27 (1947), S. 123; 31 (1951), S. 222.

402 Vgl. Heisig, Armenpolitik, 1995, S. 73. 
dürftigen - nicht nur die Sozialrentner - maßgebende typische Tatbestände festgestellt werden, die auf der Bedarfsseite die Zuerkennung eines höheren Lebensbedarfs durch Gewährung bestimmter laufender zusätzlicher Leistungen rechtfertigen“; der Ausschuß werde als mögliche Tatbestände etwa Alter oder Invalidität untersuchen und prüfen, „welches Maß der Leistungen bei gleichzeitiger Berücksichtigung der berechtigten Bedürfnisse der Hilfsbedürftigen und der öffentlichen Finanzen erreichbar erscheint" ${ }^{4}{ }^{403}$

In enger Zusammenarbeit mit dem DV legte die Sozialabteilung Anfang Juli 1951 den „Entwurf eines Gesetzes über die Änderung und Ergänzung fürsorgerechtlicher Bestimmungen“ vor, der u.a. die volle Anrechnung aller Einkünfte vorsah und solchen Hilfsbedürftigen einen Mehrbedarf zuerkannte, die über 70 Jahre alt oder schwererwerbsbeschränkt waren. ${ }^{404}$ Dieses Mehrbedarfs-Konzept bewegte sich gleichsam zwischen den beiden sozialpolitischen Extrempunkten der zeitgenössischen sozialpolitischen Leitvorstellungen: Es verabschiedete weitgehend die von Versicherungs- bzw. Versorgungsansprüchen abgeleiteten Rechtsansprüche der alten Gruppenfürsorge, machte aber auch nicht die individuelle Bedürftigkeitsprüfung zur alleinigen Leitmaxime, sondern räumte neue, wenn auch aus Bedarfstatbeständen abgeleitete Rechtsansprüche ein. Insofern war es tatsächlich dazu angetan, das unter dem Schlagwort der „Rentenkumulierung“ diskutierte Knäuel verschiedenster Sozialleistungen etwas zu entwirren, die gesetzlich statuierte Nachrangigkeit der öffentlichen Fürsorge wieder stärker zu profilieren und auch die praktische Berechnung der Hilfen in den Fürsorgeämtern zu erleichtern. Wichtiger als diese der Sicherungssystematik geschuldete „Flurbereinigung“ waren aber die aktuellen sozial- und finanzpolitischen Notwendigkeiten: Trotz kurzfristiger Erleichterungen durch das Sozialversicherungs-Anpassungsgesetz hatte sich die Lage der Rentenbezieher infolge des Preisauftriebs bis Anfang 1951 wieder dramatisch verschlechtert; SPD und Gewerkschaften forderten vehement die Erhöhung der Renten - wie auch die Freilassung dieser Erhöhungen bei der Fürsorgeberechnung. ${ }^{405}$ Die Finanzlage der Rentenversicherung war jedoch prekär, der gesamte Bundeshaushalt sogar mit einem so großen Defizit belastet, wie später während der gesamten Adenauer-Ära nicht mehr. Auch die Haushaltslage in Kommunen und Ländern war schwierig. Unter diesen Vorzeichen versuchten das vom Bundesinnenministerium sekundierte Bundesfinanzministerium auf der einen, das Bundesarbeitsministerium auf der anderen Seite, in den Verhandlungen über den Entwurf jeweils ihre Maximalpositionen durchzusetzen: völlige Anrechnung sämtlicher Renteneinkommen, möglichst niedrige Mehrbedarfssätze, keine automatischen Richtsatzerhöhungen (Finanzministerium); oder aber völlige Freilassung der Grundrente für Kriegsopfer, prozentuale Anrechnungsfreiheit bei den Sozialversicherungsrenten und indexgebundene automatische Anpassung der Richtsätze (Arbeitsministerium). Nachdem das Finanzministerium die volle Anrechnung der Sozialversicherungsrenten zur condi-

403 Vgl. NDV 31 (1951), S. 90.

${ }^{404}$ Vgl. Heisig, Armenpolitik, 1995, S. 67.

405 Zur Rentenerhöhungsdebatte 1950/51 und deren Rahmenbedingungen vgl. Hockerts, Entscheidungen, S. $171 \mathrm{ff}$. 
tio sine qua non seiner Zustimmung zum Entwurf gemacht hatte, lenkte das Arbeitsministerium schließlich ein, sofern die Sonderstellung der Kriegsopfer gewahrt würde. ${ }^{406}$

Im Bundesinnenministerium hielt man die finanziellen Auswirkungen der geplanten Reform für „nicht sehr wesentlich“, da die Neuregelungen ohnehin meist gängiger Praxis entsprächen. ${ }^{407}$ Auf kommunaler Seite gingen die Meinungen darüber jedoch auseinander und hingen entscheidend vom Herkunftsland ab: In den Ländern der ehemaligen britischen Zone, wo bisher Teile der Renten freigelassen worden waren, rechneten die zuständigen Finanzexperten mit insgesamt vertretbaren jährlichen Mehrbelastungen (Kiel: 155000 DM, Köln: 31000 DM, Düsseldorf: mindestens 570000 DM) oder im Falle Niedersachsens mit seinen besonders hohen Freibeträgen und Individualzuschlägen sogar mit Einsparungen. ${ }^{408}$ Der Bayerische Städteverband hingegen befürchtete allein für München einen jährlichen Mehrbedarf von mindestens 3 Mio. DM, und auch der Frankfurter Stadtrat Prestel rechnete mit jährlichen Mehrkosten für seine Stadt von bis zu 1 Mio. DM im Jahr, da hier ja schon bisher die Renten voll angerechnet worden waren. ${ }^{409}$ Großzügige Schätzungen über die Zahl der künftig neu in der Fürsorge zu betreuenden Kriegsbeschädigten und Sozialrentner und die fehlende Berücksichtigung der Erstattungen über die Kriegsfolgenhilfe trugen ein übriges zu diesen Prognosen bei. Das Statistische Bundesamt jedenfalls schätzte Anfang 1952 den künftigen Mehraufwand im Bundesdurchschnitt auf 5\% der bisherigen Gesamtkosten. ${ }^{410}$

Nachdem im Dezember in einer Koalitionsbesprechung weitgehende Übereinstimmung über den Gesetzentwurf erzielt worden war ${ }^{411}$, wurde er schließlich am 5. Februar 1952 vom Bundeskabinett unverändert verabschiedet. ${ }^{412}$ Der Entwurf

406 Vgl. Heisig, Armenpolitik, 1995, S.67f. Das am 11.7.1951 gegen die Stimmen der SPD verabschiedete Rentenzulagengesetz sah bereits die volle Anrechnung der Zulagen bei der Fürsorgeprüfung vor; vgl. Hockerts, Entscheidungen, S. 178f.

407 So wiedergegeben in einem Schreiben des DST-Referenten Storck an verschiedene Stadtkämmerer vom 8.10.1951, LAB, B Rep. 142-9, 1287.

$408 \mathrm{Vgl}$. die Mitteilungen des Kieler Bürgermeisters Fuchs vom 12.10.1951, des Kölner Oberstadtdirektors Syth vom 13.10.1951 und des Düsseldorfer Stadtkämmerers Reisinger vom 10.10.1951, ebenda. Der Hannoveraner Stadtkämmerer ging u.a. davon aus, daß künftig für 400-500 Kriegsbeschädigte von der Fürsorge jährlich $130000 \mathrm{DM}$ zu veranschlagen wären. Abzüglich der $85 \%$ Kriegsfolgenhilfe bliebe für Hannover also eine jährliche Mehrbelastung für diesen Personenkreis von 20000 DM. Erhebliche Einsparungen würden aber neben der vollen Anrechnung der Sozialrenten vor allem die künftigen Erstattungsansprüche gegenüber den Trägern der Rentenversicherung bringen (ca. 160000 DM pro Jahr); vgl. Müthling an DST, 9.10.1951, sowie das Schreiben des Hannoveraner Sozialamtsleiters Heinz Keese an Muthesius vom 11.10.1951, beide ebenda.

409 Vgl. Kessler, Geschäftsstelle des Bayerischen Städteverbandes an DST, 12. 10.1951; Prestel an DST, 12.10.1951; Schreiben des Münchner Sozialreferenten Hamm an Muthesius vom 12.10.1951, ebenda; für Nürnberg schätzte Marx die jährlichen Mehrausgaben hingegen auf ca. 250000 DM; vgl. Marx an Muthesius, 12.10.1951, ebenda.

410 Vgl. Vermerk des DST-Referenten von Alten vom 28.2.1952, ebenda.

411 Vgl. Heisig, Armenpolitik, 1995, S. 70.

412 Vgl. Kabinettssitzung am 5.2.1952, in: Kabinettsprotokolle, Bd. 5, S. 94f. Für den Bund rechnete der Innenminister nur mit geringen Mehrkosten. Anlaß zur Diskussion waren 
sah diverse Modifikationen und Ergänzungen der RFV und RGr. vor, ohne sie in Substanz und Aufbau grundsätzlich zu verändern. ${ }^{413}$ Anders als bei der Frage der Rückerstattungspflicht war zur Abschaffung der Freilassungsregelungen nach dem Reichsgesetz von 1941 ein förmliches Bundesgesetz erforderlich, das Votum der Bundestagsmehrheit mußte diesmal also mit ins Kalkül gezogen werden. Wohl auch deshalb wurde der Personenkreis, der von den neuen Mehrbedarfsregelungen profitieren sollte, über alte und invalide Personen hinaus erweitert, wobei man sich in der Begründung des Entwurfs pauschal auf Erfahrungen der Fürsorgepraxis berief. ${ }^{414}$ Einen Mehrbedarf von „20 v. H. des für sie maßgebenden Richtsatzes“415 sah der Entwurf vor für ältere Menschen ab 70 Jahren, für Schwererwerbsbeschränkte und für Mütter mit mindestens zwei jüngeren Kindern. Bei einem durchschnittlichen Richtsatz für Alleinstehende 1949 von 38,50 DM ${ }^{416}$ entsprachen diese 20\% (7,70 DM) also in etwa den Freilassungsbeträgen von 1941. In den genannten Fällen sollten die Fürsorgeverbände den erhöhten Bedarf nicht mehr einzeln prüfen, sondern grundsätzlich den Zuschlag gewähren. Indem der Mehrbedarf nicht als fester DM-Betrag, sondern in Relation zu den jeweiligen Richtsätzen ausgewiesen wurde, folgte der Entwurf genau dem Bundestagsbeschluß vom Februar 1950. Für ältere oder erwerbsbeschränkte Personen und für Frauen, die trotz Haushaltspflichten oder Pflegetätigkeit einem Erwerb nachgingen, war ein „angemessener Mehrbedarf“ anzuerkennen. Für Lehrlinge und Anlernlinge war - wie bereits in den meisten Ländern - der Richtsatz sogar zu verdoppeln.

Besonders begünstigt wurden auch die Zivilblinden: Ihnen wurde ohne Rückerstattungspflicht ein Mehrbedarf in Höhe des Richtsatzes für Pflege zuerkannt. Das bedeutete die allgemeine Einführung eines Blindenpflegegeldes in Höhe des Richtsatzes, das von den Blindenverbänden seit langem gefordert worden war. Anders als von diesen gewünscht, beschränkte der Entwurf dieses allerdings auf die hilfsbedürftigen Zivilblinden und betrachtete versorgungsähnliche Leistungen auch weiterhin als Sache der Länder. ${ }^{417}$

Der Mehrbedarf machte den Weg dafür frei, die bisherige Gruppenfürsorge mit Ausnahme der sozialen Fürsorgemaßnahmen für Kriegsopfer, die im wesentlichen vom Bund finanziert wurden ${ }^{418}$ - im Gesetz nun auch förmlich außer Kraft

wieder die Grundrenten: Jakob Kaiser (CDU) plädierte für einen Mehrbedarf von $100 \%$ der Grundrente, da im Bundestag ohnehin ein entsprechender Antrag zu erwarten sei. Lehr beharrte jedoch auf der Regelung des Entwurfs (50\%), unterstützt von Staatssekretär Alfred Hartmann vom BMF.

413 „Entwurf eines Gesetzes über die Änderung und Ergänzung fürsorgerechtlicher Bestimmungen“, BT, 1. Wp. 1949, Anlagen, Bd. 18, Drs. 3440, Anlage 1, S. $2 \mathrm{ff}$.

414 Vgl. ebenda, S. 10.

415 Irrtümlicherweise heißt es in der Bundestagsdrucksache bereits „von in der Regel 20 v. H.“ (Hervorhebung der Verfasserin); diese Einschränkung machte jedoch erst der Bundesrat; vgl. ebenda, S. 4, 16; ferner: NDV 32 (1952), S. 220.

416 Vgl. Bundesministerium des Innern (Hg.), Fürsorge, S. 16.

417 Vgl. BT, 1. Wp. 1949, Anlagen, Bd. 18, Drs. 3440, Anlage 1, S. 12.

418 Die jetzt wieder in Kraft gesetzten $\$ \mathbb{S} 19-32$ RGr. über die soziale Fürsorge für Kriegsopfer waren bisher nur durch Verwaltungsvorschriften zu den $\mathbb{\$} \$ 25-27$ BVG für anwendbar erklärt worden. 
zu setzen. In Zukunft sollte jede Rentenleistung in vollem Umfang bei der Fürsorgebemessung berücksichtigt werden, bis auf eine wichtige Ausnahme: Bei hilfsbedürftigen Kriegsbeschädigten waren monatlich 50\% der Grundrente, mindestens jedoch $10 \mathrm{DM}$ als Mehrbedarf anzuerkennen - also freizulassen. Diese Regelung war ein Kompromiß, der von der Bundesregierung mit der unklaren Zweckbestimmung der Grundrente nach dem BVG (Entschädigung für zusätzlichen Aufwand oder allgemeiner Beitrag zum Lebensunterhalt) begründet wurde. Nach Darlegung der Bundesregierung brächte die vorgesehene Regelung für die meisten hilfsbedürftigen Kriegsbeschädigten eine deutliche Verbesserung, denn fast überall wurde die Grundrente entweder ganz oder bis auf 6 DM bzw. 7 DM angerechnet. ${ }^{419}$ Die Renten der Kriegshinterbliebenen allerdings waren voll auf die Fürsorge anzurechnen.

Mit Abschaffung der Gruppenfürsorge sollten nun für alle Hilfsbedürftigen jeweils einheitliche, „den örtlichen Lebensverhältnissen angepaßte Richtsätze“ gelten. Laut Regierungsbegründung werde dadurch „nicht nur eine Gerechtigkeit der Leistung“ gesichert, sondern auch „eine einheitliche Gestaltung der Richtsatzpolitik der Länder und damit deren Vergleichbarkeit“420. Um diesen Hebel von Bundesseite nun tatsächlich ansetzen zu können, sollten die RGr. um eine Bestimmung ergänzt werden, wonach der Bundesinnenminister zusammen mit dem Bundesfinanz- und dem Bundesarbeitsminister Verwaltungsvorschriften über den Aufbau der Richtsätze erlassen konnte. Eine Verpflichtung, auch die kommunalen Spitzenverbände an der Ausarbeitung dieser Vorschriften zu beteiligen, konzedierte die Bundesregierung diesen jetzt wie auch später nicht.

Ende Februar 1952 machte der Bundesrat nur eine Reihe von Änderungsvorschlägen zugunsten der Hilfsbedürftigen, obwohl die Grundtendenz des Entwurfs vielen bisherigen Länderregelungen widersprach. Er schlug u.a. vor, die Altersgrenze für den Mehrbedarf auf 65 Jahre herabzusetzen und die Unfallrentner den Kriegsbeschädigten gleichzustellen, da auch deren Schädigung vergleichbar sei. Allerdings sollte der Mehrbedarf für alte Leute etc. nicht generell, sondern „in der Regel“ 20\% des jeweiligen Richtsatzes betragen, da auch die Gewährung eines geringeren Mehrbedarfs möglich sein müsse. ${ }^{421}$ Ferner wollte der Bundesrat auch selbst auf die stärker zentralisierte Lenkung der Richtsätze Einfluß nehmen und forderte für die geplanten Verwaltungsvorschriften über die Richtsätze ein $\mathrm{Zu}$ stimmungsrecht.

Die Bundesregierung wollte dieses Recht konzedieren, lehnte jedoch die Herabsetzung der Altersgrenze ebenso ab wie die Gleichstellung der Unfallverletzten; dabei dürften die finanziellen Bedenken der kommunalen Spitzenverbände gegen eine zusätzliche Ausweitung des begünstigten Personenkreises maßgeblich gewesen sein, die über die Kriegsfolgenhilfe auch den Bund treffen müßte. ${ }^{422}$ Tat-

419 BT, 1. Wp. 1949, Anlagen, Bd. 18, Drs. 3440, Anlage 1, S. 11.

420 Ebenda, S. 7.

421 Vgl. „Änderungsvorschläge zu dem Entwurf eines Gesetzes über die Änderung und Ergänzung fürsorgerechtlicher Bestimmungen“"vom 29.2.1952; ebenda, S. 16.

422 Vgl. die Stellungnahme der Bundesregierung zu den Änderungsvorschlägen des Bundesrates, ebenda, Anlage 3; ferner NDV 32 (1952), S. 220. 
sächlich waren Ende 1951 nur rund 5\% aller Fürsorgeempfänger zwischen 65 und 70 Jahren alt ${ }^{423}$, eine Herabsetzung der Altersgrenze hätte also keine nennenswerten Mehrkosten gebracht, zumal es sich in der Regel um zusätzlich - und u.U. nur vorübergehend - zu Unterstützende gehandelt haben dürfte. Der Einschränkung der Mehrbedarfsgewährung auf „in der Regel 20 v.H.“ stimmte die Bundesregierung demgegenüber zu. Bemerkenswerterweise stieß sie damit jedoch auf den Widerspruch des DST und des DLT: Der starre Mehrbedarf solle nämlich „sicherstellen, daß überall - und damit auch im Bereich von Gemeinden, bei denen eine weniger fortschrittliche oder weniger soziale Haltung vorliegt - die [...] erhöhte Unterstützung auch tatsächlich gezahlt" werde. ${ }^{424}$ Wenn sich die kommunalen Spitzenverbände hier zu Anwälten der Hilfsbedürftigen machten, dann nicht zuletzt aus der Furcht, eine Verwässerung dieser für die Rentenbezieher relevanten Mehrbedarfsregelungen könne schließlich das ganze Gesetz zum Scheitern bringen. So begrüßte der DST-Sozialausschuß den Regierungsentwurf auch als „beträchtlichen sozialpolitischen Fortschritt“, der eine "gerechte und individuelle Bemessung der Leistungen“ ermögliche; sofern nicht künftig wieder neue Freilassungen beschlossen würden, sei auch die mindestens fünfprozentige Kostensteigerung noch zu vertreten. ${ }^{425}$

Auf Kritik stieß der Entwurf hingegen vor allem bei den Kriegsopferverbänden, dem DGB und auch bei der SPD. In einer Umfrage der DGB-Zeitschrift „Soziale Sicherheit“ vom Frühling 1952 forderten sie die volle Freilassung der Grundrenten der Kriegsopfer und eine analoge Regelung für Unfallrentner; die Grundrente sei eben keine Versorgungsrente, sondern diene allein zum Ausgleich des durch Beschädigung oder den Tod des Ernährers notwendigen Mehrbedarfs. ${ }^{426}$ Einig war man sich auch über eine Besserstellung der Sozial- und Kleinrentner, da deren eigene Altersvorsorge angemessen berücksichtigt werden müßte. Der „Verband der Kriegsbeschädigten, Kriegshinterbliebenen und Sozialrentner“ $(\mathrm{VdK})$ und der Reichsbund sowie Walter Henkelmann von der DGB-Sozialabteilung wollten deutlich erhöhte Freilassungen (DGB) bzw. Richtsätze (Reichsbund), plädierten also für eine Aufrechterhaltung der Gruppenfürsorge. Die SPDAbgeordnete Lisa Korspeter, Mitglied im Bundestagsfürsorgeausschuß, wollte dagegen das neue Mehrbedarfskonzept mittragen, wenn die Altersgrenzen herabgesetzt und die Definition der Erwerbsunfähigkeit abgemildert würde.

Am 18. Juli 1952 überwies der Bundestag den Entwurf ohne Aussprache an den Ausschuß für Fragen der öffentlichen Fürsorge ${ }^{427}$, der ihn acht Monate lang beriet. Mit Ausnahme einer etwas spitzfindigen Sonderregelung für die Kriegsbe-

${ }^{423}$ Vgl. Bundesministerium des Innern (Hg.), Fürsorge, S. 21.

424 Gemeinsame Eingabe des DST und des DLT beim Bundestagsausschuß für Fragen der öffentlichen Fürsorge vom 6. 5.1952, LAB, B Rep. 142-9, 1287.

425 Zitat s. Protokoll der Sitzung des DST-Sozialausschusses am 4./5.4.1952, ferner Eingabe des DLT und des DST vom 6.5.1952, ebenda; ähnlich positiv auch die abschließende Bewertung des neuen Gesetzes durch den Vorsitzenden des DLT-Sozialausschusses Schmerbeck, in: Die Selbstverwaltung 7 (1953), S. 221.

426 Vgl. Soziale Sicherheit 1 (1952), S. 118ff.; ferner die Änderungsvorschläge des DGB zum Entwurf eines Gesetzes [...] vom 17.11.1952, LAB, B Rep. 142-9, 1287.

427 Vgl. Sitzung des Bundestages am 18. 7.1952, BT, 1. Wp. 1949, Sten. Ber., Bd. 12, S. 10211. 
schädigten machte sich der Ausschuß einmütig die Zielsetzung des Entwurfs zu eigen, statt Freilassungen Mehrbedarfstypen einzuführen. ${ }^{428}$ Auch der Reichsbund hatte in einem „Sozialpolitischen Sofortprogramm für das Fürsorgerecht" vom 20. März $1953^{429}$ schließlich die Einführung des Mehrbedarfs prinzipiell akzeptiert und nur für die Kriegsopfer die Forderung nach voller Freilassung der Grundrente aufrechterhalten. Da aber in Anbetracht der bevorstehenden Bundestagswahlen keine Seite die Rentner völlig verprellen wollte, beschloß der Ausschuß doch eine Reihe von Verbesserungen für diese und andere Hilfsbedürftige, die im wesentlichen auch Eingang in das spätere Gesetz fanden: Die Altersgrenze wurde auf 65 Jahre herab- (Art.IV), und der Mehrbedarf für alte und schwerbeschädigte Personen in jedem Falle auf 20\% des Richtsatzes festgesetzt (Art. IV). Deutlich erhöht wurden schließlich die Mehrbedarfssätze für die Zivilblinden (Art.IV), um sie gegenüber einzelnen Länderregelungen nicht schlechter zu stellen. ${ }^{430}$

Einen komplizierten Kompromiß fand der Ausschuß für die Kriegsbeschädigten: In den allgemeinen Teil der RGr. wurde ein Passus eingefügt, wonach die neuen Mehrbedarfsbestimmungen die Vorschriften des Bundesversorgungsgesetzes über die Gewährung von weitergehenden Leistungen der sozialen Fürsorge nicht berührten, die in den $\mathbb{S}$ 19-32 RGr. geregelt wurde (Art. IV). Bei Beschädigten sollte jetzt „ein Mehrbedarf als Ausgleich für die Folgen der Schädigung in Höhe der Grundrente" anerkannt werden (Art.IV a). Das bedeutete letzten Endes: Bei denjenigen Beschädigten, die auch ein Anrecht auf soziale Fürsorge hatten, weil ihre Notlage durch die „Dienstbeschädigung“ verursacht war, war die Grundrente freizulassen. Das galt nicht für die Hinterbliebenen und die (kleine) Zahl derjenigen, die keinen Anspruch auf Sozialfürsorge hatten. ${ }^{431}$ Wenn das auch von der Berichterstatterin Niggemeyer im Bundestag nicht so deutlich gesagt wurde: Zumindest für die Kriegsbeschädigten selbst hatte der Ausschuß im Vorfeld der Bundestagswahl doch eine Ausnahme vom Grundsatz der vollen Anrechnung gemacht, eine Ausnahme, die im Rahmen der Kriegsfolgenhilfe im wesentlichen vom Bund zu finanzieren war. Die von der SPD gewünschte Gleichstellung der Unfallrentner und NS-Opfer hingegen wurde von der Ausschußmehrheit abgelehnt. Dem in der Frage der Sozialrenten kompromißbereiten Reichsbund kam der Ausschuß bei einem alten Anliegen dann weit entgegen: Wie auch von den SPD-Vertretern gefordert, wurde die 1939 suspendierte Verpflichtung zur Beteiligung von Betroffenen bzw. ihrer Organisationen bei der Aufstellung von Richtsätzen und Richtlinien sowie bei Einspruchsverfahren (ehemaliger $\mathbb{3}$ a RFV) in formalrechtlich modifizierter Form wieder eingeführt (Art. I).

Als weitere zukunftsweisende Neuerung zählte der Ausschuß neben der Erwerbsbefähigung jetzt auch die Ausbildung für einen Beruf zu den Pflichtleistun-

${ }^{428}$ Vgl. Mündlicher Bericht des Ausschusses für Fragen der öffentlichen Fürsorge vom 21.5.1953, BT, 1. Wp. 1949, Anlagen, Bd.23, Drs. 4371. Darauf beziehen sich im Folgenden die Artikel-Angaben.

429 ADW, HGSt, SP-S XXXIX 7: 0-1/1.

430 Vgl. Abg. Niggemeyer (CDU) am 12.6.1953, BT, 1. Wp. 1949, Sten. Ber., Bd. 16, S. 13404.

431 Vgl. die Erläuterungen von Gottschick/Keese, Fürsorgeänderungsgesetz, S. 93f., $108 \mathrm{f}$. 
gen für Minderjährige, Körperbehinderte und Volljährige, die durch die Kriegsfolgen keine Ausbildung hatten erlangen oder abschließen können (Art. Ib). Innerhalb des Ausschusses zunächst umstritten blieb die Regelung der Richtsatzfestsetzung: Reichsbund und Sozialdemokraten forderten, daß die jeweiligen Lebenshaltungskosten zwingend bei der Festsetzung berücksichtigt werden müßten. Das Bundesinnenministerium und die Ausschußmehrheit meinten jedoch, daß auch die Formulierung des Regierungsentwurfs die Anpassung der Richtsätze an die wirtschaftliche Entwicklung garantiere. ${ }^{432}$ Mit einigen weiteren Änderungen wurde der Gesetzentwurf in seiner Gesamtheit schließlich einstimmig vom Ausschuß angenommen.

In der zweiten und dritten Lesung im Bundestag am 12. Juni 1953 konnte die SPD mit den Stimmen der kleineren Parteien dann doch noch gegen die CDU/CSU ihren Antrag durchsetzen, hilfsbedürftigen Unfallverletzten und rentenberechtigten Verfolgten des NS-Regimes einen Mehrbedarf in Höhe einer vergleichbaren Versorgungs-Grundrente zuzugestehen ${ }^{433}$. In seiner Gesamtheit wurde das Gesetz dann allerdings bei einigen Enthaltungen von sämtlichen Parteien beschlossen. Einen Tag zuvor jedoch hatte der Bundestag die Gleichstellung der Unfallverletzten mit den Kriegsbeschädigten im Rahmen der Arbeitslosenfürsorge abgelehnt, so daß jetzt zum gleichen Problem zwei unterschiedliche Beschlüsse vorlagen. ${ }^{43}$ Innenminister Lehr schlug daher die Anrufung des Vermittlungsausschusses vor mit dem Ziel, das Fürsorgeänderungsgesetz dem Beschluß zur Arbeitslosenfürsorge anzupassen. ${ }^{435}$ Der vom Finanzminister lebhaft unterstützten Vorlage stimmte das Bundeskabinett am 23. Juni zu. Auch der Bundesrat wandte sich in Widerspruch zu seiner ursprünglichen Stellungnahme jetzt gegen die Bevorzugung der Unfallverletzten und beschloß gegen die Stimmen der SPD-regierten Länder Hamburg, Hessen und Niedersachsen deshalb ebenfalls, den Vermittlungsausschuß anzurufen. ${ }^{436}$ Außerdem forderte die Mehrzahl der Länder u.a. die ersatzlose Streichung des Art. I, da die Besetzung der entsprechenden Gremien erfahrungsgemäß schwierig sei und ihre Einschaltung die Verantwortlichkeiten verwische.

Schließlich wurde auf Vorschlag des Vermittlungsausschusses im Sinne der Bundesregierung der erhöhte Mehrbedarf begrenzt auf schwerbeschädigte Unfallrentner, deren Hilfsbedürftigkeit mit ihrer Schädigung zusammenhing. ${ }^{437}$ Die Mitwirkung der Hilfsbedürftigen wurde erheblich eingeschränkt: bei der Aufstellung der Richtlinien und Richtsätze auf ein Anhörungsrecht, bei Einspruchsverfahren auf eine beratende Beteiligung. Darüber hinaus wurde die Vertretung durch Wohlfahrtsverbände und Organisationen anderer Sozialleistungsempfänger

432 Vgl. Heisig, Armenpolitik, 1995, S. 73.

433 Vgl. Bundestagssitzung am 12.6.1953; BT, 1. Wp. 1949, Sten. Ber., Bd.16, S. $13410 f f$.

434 Vgl. Sitzung am 11.6.1953; ebenda, S. 13309.

435 Vgl. Kabinettssitzung am 23.6.1953, in: Kabinettsprotokolle, Bd. 6, S. 359.

436 Vgl. Sitzung am 26.6.1953, BR 1953, Sten. Ber., S.33ff., sowie die entsprechende Begründung, BT, 1. Wp. 1949, Anlagen, Bd.25, Drs. 4616.

437 Gottschick/Keese, Fürsorgeänderungsgesetz, S. 94, schätzten die zahlenmäßige Auswirkung ohnehin als gering ein, da die Renten der meisten Unfallrentner und NS-Opfer den fürsorgerischen Bedarf überstiegen. 
erleichtert. ${ }^{438}$ In dieser modifizierten Fassung konnte das „Fürsorgeänderungsgesetz“ (FÄG) schließlich am 20. August 1953 im Bundesgesetzblatt veröffentlicht werden. ${ }^{439}$ Einen Tag zuvor hatte der Bundesinnenminister die zuständigen Länderminister schriftlich gedrängt, das Gesetz bereits vor seinem offiziellen Inkrafttreten zum 1.Oktober, nämlich rückwirkend ab 15. August, anzuwenden, damit sich die Leistungsverbesserungen möglichst frühzeitig auswirken könnten. ${ }^{440}$ Dieser Aufforderung, die weniger im Blick auf das Wohl der Fürsorgebezieher als auf die Bundestagswahlen geschah, konnten die Länder nach eigenem Bekunden aber nicht Folge leisten, da ihnen die Anwendung ab 1. Oktober schon schwierig genug fiel. ${ }^{441}$

Oberregierungsrat Anton Oel von der Sozialabteilung rechnete damit, daß etwa 40\% aller Fürsorgeempfänger Anspruch auf Mehrleistungen nach dem neuen Gesetz haben würden. ${ }^{42}$ Tatsächlich aber ist die Wirkung des Fürsorgeänderungsgesetzes für die betroffenen alten, behinderten und andere Fürsorge benötigenden Menschen mangels aussagekräftiger Statistiken an dieser Stelle kaum zu beschreiben. ${ }^{443}$ Finanzpolitisch brachte das Gesetz jedenfalls klare Verhältnisse insofern, als die Abrechnung der Kriegsfolgenhilfe nicht mehr durch unterschiedliche Freilassungsbestimmungen ungleich gehandhabt wurde. Dies war aus Sicht des Bundes umso notwendiger, als nach dem kurz zuvor verabschiedeten "Fremd- und Auslandsrentengesetz" vom 7. August 1953 die Vertriebenen und Flüchtlinge nun bundesweit die gleichen, vom Bund zu finanzierenden Renten-

438 Vgl. den mündlichen Bericht des Vermittlungsausschusses vom 2.7.1953, BT, 1. Wp. 1949, Anlagen, Bd. 25, Drs. 4639. Der Bundestag nahm den Vorschlag des Vermittlungsausschusses in seiner Sitzung am 3.7.1953 an, vgl. ebenda, Sten. Ber., Bd.17, S.14141, der Bundesrat am 17.7.1953, vgl. BR 1953, Sten. Ber., S. 18. Zur Bewertung des neuen $₫ 3$ a vgl. Oel, Beteiligung.

439 BGBl. I S. 967.

$440 \mathrm{Vgl}$. Staatssekretär Bleek an die Sozial- bzw. Innenministerien der Länder, 19.8.1953, sowie die einschlägigen Antwortschreiben, LAB, B Rep. 142-9, 1287.

$441 \mathrm{Zu}$ den deshalb Anfang 1954 vom BMI herausgegebenen Richtlinien über die Höhe des Mehrbedarfs bei geringfügigem Erwerb und ein Rundschreiben betr. Kumulierungen bestimmter Mehrbedarfe s. Jehle, Fürsorgerecht, S.352f.; vgl. NDV 34 (1954), S. 108f., 142ff., 243.

$442 \mathrm{Vgl}$. Oel, Fürsorge-Änderungsgesetz, S. 281f.

443 So wissen wir z.B., daß die Zahl der von der Fürsorge laufend zusätzlich unterstützten Sozialrentner bis 1955 weiter anstieg auf 188000 (1950: 136000), ihr Anteil an allen Fürsorgeempfängern sogar von rund 20\% (Juni 1950) über fast 27\% (im September 1953, also unmittelbar vor Inkrafttreten des FÄG) auf knapp 30\% (März 1955). Daraus jedoch zu folgern, die neuen Mehrbedarfsregelungen wären für diese Gruppe günstiger als die alten Freilassungsbestimmungen, ist nicht möglich: Zwischen 1950 und 1955 stieg die Zahl der Sozialrentner insgesamt von 4,3 Mio. auf 6,6 Mio., der Anteil derjenigen, die zusätzlich Fürsorgeunterstützung erhielten, verminderte sich sogar minimal auf 2,9\%; außerdem blieb der Anteil der Hilfsbedürftigen über 65 Jahre in der Fürsorge annähernd gleich (1951: 17\%; 1955: 19\%). Der deutlich gestiegene Anteil der Rentner an allen Fürsorgeempfängern belegt vielmehr die Entlastung der öffentlichen Fürsorge durch günstige Konjunktur (Ausscheiden anderer Hilfsbedürftigengruppen, wodurch die zahlenmäßige Bedeutung der Rentner relativ steigt) und andere sozialpolitische Maßnahmen (Anerkennung der Rentenansprüche der Heimatvertriebenen, die jetzt allenfalls zusätzlich zu unterstützen waren); vgl. Bundesministerium des Innern (Hg.), Fürsorge, S. 21, 28f.; Schäfer, Rolle, S. 203ff. 
ansprüche wie die „Einheimischen“ erhielten; deren teilweise Freilassung hätte den Bund also doppelt belastet. Aus dem verstärkten Interesse an einer einheitlicheren Fürsorgebemessung resultierte auch die im Änderungsgesetz ausgesprochene Ermächtigung, eine Verordnung über den Aufbau der Richtsätze zu erlassen. Damit schuf das Gesetz die rechtliche Grundlage für das 1955 installierte Warenkorb-Modell zur Bestimmung des fürsorgegestützten Existenzminimums. Sie war auch der Preis dafür, daß der Bundestag künftig nicht mehr durch Freilassungsbeschlüsse ohne weiteres in die kommunale Domäne der Fürsorgebemessung einbrechen konnte.

Darüber hinaus bildete das Mehrbedarfskonzept eine wichtige Teilstufe auf dem Weg zum BSHG, in das die Mehrbedarfskriterien fast unverändert übernommen wurden. Mit dem „Schönheitsfehler“ der Sonderregelung für die Kriegsbeschädigten, die im Sommer 1957 dann doch auf die Hinterbliebenen ausgedehnt wurde ${ }^{444}$, markierte das Fürsorgeänderungsgesetz mit dem Übergang vom Kausal- zum Finalprinzip formalrechtlich den endgültigen Abschied von der Gruppenfürsorge, die seit den Jahren des Ersten Weltkriegs ein wesentliches Kennzeichen deutscher Wohlfahrtspflege gewesen war. Diese Abkehr von einer primär nach sozialen Kriterien ausdifferenzierten Fürsorge war politisch vor allem deshalb möglich geworden, weil die früher bevorzugten Gruppen jetzt, im beginnenden Wirtschaftsaufschwung, durch höhere bzw. neue Versicherungs- und Versorgungsleistungen außerhalb der Fürsorge besser abgesichert wurden als in den Notzeiten der Weimarer Republik. ${ }^{445}$

\section{Der Rechtsanspruch auf Fürsorge: das Urteil des Bundesverwaltungsgerichts von 1954}

Die Ausweitung staatlich garantierter Rechtsansprüche auf Sozialleistungen gilt als ein wichtiges Merkmal der sozialpolitischen Entwicklung nicht nur in Deutschland und ist gleichzeitig Gradmesser für die „Modernität“ eines sozialen Sicherungssystems. ${ }^{446}$ Verglichen mit Versicherung und Versorgung war die Rechtsstellung des Sozialleistungsempfängers innerhalb der Fürsorge traditionell schwach und damit nach dem Zweiten Weltkrieg wesentlicher Grund für das schlechte Image der Fürsorge. Diese Schwäche gründete auf der tradierten Auffassung über die Klientel der (Armen-)Fürsorge, die keine wie auch immer definierte Vorleistung (Beitragszahlung, besondere Dienste für die Allgemeinheit) erbracht habe und daher auch keine Ansprüche auf Gegenleistung erheben könne. Hilfsbedürftigkeit galt lange vor allem als Störung der öffentlichen Ordnung, der Arme, so das Bundesamt für das Heimatwesen 1901, war „nur der Gegenstand

${ }^{444}$ Mit dem „Gesetz zur Änderung der Reichsgrundsätze über Voraussetzung, Art und Maß der öffentlichen Fürsorge" vom 4.7.1957, BGBl. I S.693, wurde im Vorfeld der Bundestagswahlen auch den Hinterbliebenen ein Mehrbedarf in Höhe ihrer Grundrente zuerkannt und so der besonders schwierigen Situation vieler Kriegerwitwen und ihrer Kinder Rechnung getragen.

445 Vgl. Tennstedt, Fürsorgegeschichte, S. 98.

446 Vgl. u.a. - in Anlehnung an ein Diktum Ernst Schellenbergs - Sund, Entwicklung. 
der den Armenverbänden im öffentlichen Interesse auferlegten Unterstützungspflicht“447. Außerdem hatte die Verrechtlichung eine Standardisierung sozialer Leistungen zur Folge und geriet damit in Widerspruch zum Individualisierungsprinzip der Fürsorge, das idealiter auf die jeweiligen Bedürfnisse des Hilfesuchenden zu reagieren hatte und sich näherer juristischer Definition entzog.

Anders als während des Kaiserreichs behielt der Fürsorgeempfänger in der Weimarer Republik zwar das allgemeine Wahlrecht, und die neue Reichsverfassung statuierte u.a. für kinderreiche Familien einen „Anspruch auf ausgleichende Fürsorge" (Art. 119) ${ }^{448}$. Als der Fürsorgefachmann Friedrich Diefenbach in einer im Auftrag des DV erstellten Schrift 1920 forderte, dem Hilfsbedürftigen einen Anspruch auf Unterstützung einzuräumen und dadurch die Armengesetzgebung an das Sozialversicherungsrecht anzunähern, stand er jedoch weitgehend allein. ${ }^{449}$ RFV, RGr. und deren Änderungen und Ausführungsvorschriften stärkten durch konkrete Hilfenkataloge, Richtsatzdefinition etc. zwar die Rechtsstellung des Hilfeempfängers, einen generellen subjektiv-öffentlichen Rechtsanspruch auf Fürsorge hingegen statuierten sie nicht. Zeitgenössische Rechtsprechung und gängige Rechtsmeinung betrachteten Fürsorge als objektive Pflicht des Staates, verneinten unter Hinweis auf die historische Entwicklung wie auch die Notwendigkeit weiter behördlicher Ermessensräume aber einen subjektiven Rechtsanspruch. ${ }^{450} \mathrm{Die}$ nationalsozialistische Ideologie der "Volksgemeinschaft" setzte an die Stelle der Solidarität der Gesellschaft zugunsten der Schwachen das Postulat größtmöglicher Opferbereitschaft des einzelnen für das Volk und ließ damit schon theoretisch keinen Raum mehr für individuelle Rechtsansprüche gegenüber der Allgemeinheit ${ }^{451}$ - von der fürsorgerischen Praxis ganz zu schweigen.

Obwohl die Rechtsgrundlagen der öffentlichen Fürsorge 1949 vorerst unverändert geltendes Bundesrecht wurden, anerkannten jetzt immer mehr Verwaltungsgerichte einen subjektiv-öffentlichen Rechtsanspruch auf öffentliche Fürsorge und beriefen sich dabei auf entsprechende Implikationen der Landesverfassungen und des Bonner Grundgesetzes. Den Anfang machte im März 1949 der Verwaltungsgerichtshof Bayerns, dessen stark sozialpolitisch ausgerichtete Verfassung u.a. jedem arbeitsunfähigen oder nicht vermittelbaren Einwohner ein „Recht auf Fürsorge“ (Art. 168 Abs. 3 BayVerf.) zuerkannte: Es sei mit „den Grundforderungen des modernen Rechts- und Sozialstaates [...] nicht zu vereinbaren, die Hilfsbedürftigen in die Rolle von Menschen zurückzudrängen, deren Anwartschaft auf

447 Entscheidung des Bundesamtes für das Heimatwesen vom 22.6.1901, zitiert in: NDV 34 (1954), S. 133.

448 Auch der lange umstrittene $\int 1$ des RJWG von 1922 sprach jedem deutschen Kind ein „Recht auf Erziehung“ zu, doch die offizielle Begründung erklärte, daß es sich hierbei um eine programmatische Verpflichtung des Staates und „kein klagbares Recht“ des Kindes handele; vgl. Hasenclever, Jugendhilfe, S. 63.

449 Eine ähnliche Mindermeinung war bereits 1905 von Rudolf Schwander vertreten worden, vgl. insgesamt NDV 34 (1954), S.133f.; Wolfrum, Rechtsstellung, S. 32ff.; Sachße/ Tennstedt, Geschichte, Bd.2, S. 143f.

450 Zur rechtlichen Entwicklung seit den zwanziger Jahren siehe knapp Stolleis, Quellen, S. 23f. Literaturhinweise in: NDV 28 (1948), S. 120.

${ }^{451} \mathrm{Vgl}$. Sachße/Tennstedt, Geschichte, Bd. 3, S. $51 \mathrm{ff}$. 
Fürsorge sich nur indirekt als Reflexwirkung daraus ergäbe, daß die Fürsorgeverbände gegenüber dem Staat zur Bekämpfung der Armut verpflichtet sind. “452 Erfahrungen mit der neuen demokratischen Staatsform wie auch mit einer andersartigen Fürsorgeklientel trugen hier erste Früchte.

Einen Rechtsanspruch auf Fürsorge bejahten nun immer mehr oberste Verwaltungsgerichte; sie stützten sich insbesondere auf die grundgesetzliche Garantie des Rechts auf Leben und körperliche Unversehrtheit (Art. 2 Abs. 2 GG), die verfassungsmäßige Rechts- und Sozialstaatlichkeit der Bundesrepublik (Art. 20 und 28 GG) sowie die Rechtsweggarantie (Art. 19 Abs. 4 GG). ${ }^{453}$ Begünstigt wurde die weniger mit den Interessen der Fürsorgeämter konforme Rechtsprechung durch die Unabhängigkeit der Verwaltungsgerichte, die das bis 1939 zuständige Bundesamt für das Heimatwesen nie für sich beanspruchen konnte. ${ }^{454}$

Unter Rechtswissenschaftlern wie innerhalb des DV herrschten zunächst unterschiedliche Ansichten. Im NDV wurde im Sommer 1948 gemutmaßt, daß „ein solches, durch Klage vor dem Verwaltungsgericht verfolgbares Recht auf Fürsorge in Notzeiten zu einer erheblichen Verstärkung des von der Gesamtheit der Hilfsbedürftigen ausgehenden Druckes führen“ werde. ${ }^{455}$ Nach Inkrafttreten des Grundgesetzes plädierten jedoch die DV-Vorstandsmitglieder Prestel und Treibert auf dem Fürsorgetag 1949 für einen subjektiven Rechtsanspruch in allgemeiner Form. ${ }^{456}$ Anfang 1951 ging auch Muthesius davon aus, daß angesichts der Rechtsprechung ein „Rechtsanspruch auf Unterstützung“ in ein neues Bundesfürsorgegesetz aufgenommen werden müsse, freilich mit dem Zusatz: „Niemand darf unter Berufung auf das Bundesfürsorgegesetz seine eigenen Pflichten vernachlässigen“. ${ }^{457}$ Kritiker befürchteten allerdings, daß Verwaltungsrichter auch den behördlichen Ermessensraum und damit insbesondere die Höhe der Richtsätze zum Gegenstand ihrer Rechtsprechung machen würden. ${ }^{458}$ Insgesamt blieben DV und kommunale Spitzenverbände abwartend, wenn auch die zuständige Arbeitsgruppe auf dem Fürsorgetag 1951 bereits einräumte, daß der allgemeine Trend der Rechtsprechung von seiten der Fürsorgegesetzgebung „kaum rückgängig zu machen“ sei. ${ }^{459}$ Einen Rechtsanspruch auf eine von den Gruppenhilfen befreite, tatsächlich nur mehr individuell tätige Fürsorge hatte auch Ludwig Preller auf dem Fürsorgetag nicht gefordert, diesen sah sein Neuordnungsprogramm vielmehr ausdrücklich für die gruppenbezogenen „sozialpolitischen Leistungen“ einschließlich einer allgemeinen Grundrente vor. ${ }^{460}$

452 Die Öffentliche Verwaltung 1949, S.375; dazu NDV 29 (1949), S. 223f.; vgl. auch Oel, Recht, S. 119; Susanne Hauser, Geschichte, S. 212, 225.

453 Vgl. NDV 30 (1950), S. 269ff.; Manderschied, Rechtsanspruch; NDV 34 (1954), S. 133ff.; Oestreicher, Entwicklung, S. 68ff.

454 Vgl. den knappen Überblick bei Flamm, Die Verwaltung der behördlichen Sozialarbeit, S. 96ff.; ferner Unruh, Verwaltungsgerichtsbarkeit.

455 NDV 28 (1948), S. 121.

456 Vgl. Prestel, Gebiet, S. 33; Treibert, Gebiet, S. 55.

457 Muthesius am 22.1.1951 vor dem Fachausschuß I, NDV 32 (1952), S. 31.

458 Vgl. NDV 30 (1950), S. 269ff.; Schallock, Recht; NDV 34 (1954), S. $358 f f$.

459 Vgl. NDV 31 (1951), S. 339.

460 Vgl. ebenda, S. 325. 
Das Fürsorgeänderungsgesetz ging ebenfalls noch nicht den entscheidenden Schritt, wenn auch die Mehrbedarfsregelungen den Rechtsschutz des Hilfsbedürftigen praktisch verstärkten. Muthesius allerdings forderte in Konsequenz seines Konzepts einer staatlichen Einkommenshilfe für die künftige große Reform nun entschieden einen Rechtsanspruch auf die laufenden Fürsorgeunterstützungen, „damit das so schwerwiegende [...] Phänomen der Abhängigkeit des einzelnen Menschen von anderen Menschen gemildert wird, um zur Wahrung der Würde des einzelnen Menschen in seiner Familie und der Gesellschaft beizutragen". 461

Anfang 1954 setzte dann auch der Fachausschuß I des DV das Thema „Rechtsanspruch" auf seine umfangreiche Arbeitsliste ${ }^{462}$, doch der entscheidende Anstoß für ein allgemeines Umdenken kam von außen: In einem seiner frühen Urteile anerkannte das Bundesverwaltungsgericht am 24.Juni 1954 einen Rechtsanspruch auf Pflichtleistungen der öffentlichen Fürsorge. ${ }^{463}$ Die bisher gängige Ablehnung eines Rechtsanspruchs habe nicht an eine ausdrückliche Bestimmung des Fürsorgerechts angeknüpft, „sondern beruhte auf hergebrachten sozialethischen Vorstellungen. [...] Spätestens seit dem Inkrafttreten des Grundgesetzes ist die frühere Auffassung nicht mehr haltbar.“ Ausgehend von den „Leitgedanken des Grundgesetzes“ stellte das Gericht daher fest: „Der Einzelne ist zwar der öffentlichen Gewalt unterworfen, aber nicht Untertan, sondern Bürger. Darum darf er in der Regel nicht lediglich Gegenstand staatlichen Handelns sein. Er wird vielmehr als selbständige sittlich verantwortliche Persönlichkeit und deshalb als Träger von Rechten und Pflichten anerkannt. Dies muß besonders dann gelten, wenn es um seine Daseinsmöglichkeit geht." ${ }^{464}$

Nach rechtswissenschaftlicher Auslegung war das Urteil von 1954 ein „Markstein“ für die Entwicklung der Fürsorge zur Sozialhilfe ${ }^{465}$ und wird als „Wendung von der staatsinternen Bindung zum subjektiv-öffentlichen Recht" interpretiert. ${ }^{466}$ Die Bedeutung für die fürsorgerische Praxis wurde jedoch schon sehr bald u.a. im NDV dahingehend relativiert, daß der Rechtsanspruch zunächst nur besage, „daß der Fürsorgesuchende das Verwaltungsgericht anrufen kann mit der Behauptung, daß die Fürsorgebehörde bei der Ablehnung seines Fürsorgeantrages rechtswidrig gehandelt" habe, einen Anspruch auf eine bestimmte Fürsorgeleistung garantiere er nicht. 467 Ein anderer Kommentar im NDV bewertete dann auch die "sozial-ethische“ Bedeutung des Urteils höher als dessen praktische Konsequenzen, da sich an „Voraussetzungen, Art und Maß“ der behördlichen

461 Muthesius auf dem Fürsorgetag 1953; NDV 34 (1954), S. 74.

462 Vgl. Niederschrift über die Sitzung des Fachausschusses I am 4.2.1954, LAB, B Rep. 142-9, 1256.

463 Der Leitsatz des Urteils lautete: „Soweit das Gesetz dem Träger der Fürsorge zugunsten des Bedürftigen Pflichten auferlegt, hat der Bedürftige entsprechende Rechte“, BVerwGe I, S. 159.

464 Urteilsbegründung ebenda, S. 159-163, hier S. $160 f$.

465 Vgl. Giese, 25 Jahre, S. 254.

466 So der Rechtshistoriker Michael Stolleis, Quellen, S.24; ähnlich Sund, Entwicklung, S. 155.

467 NDV 34 (1954), S. 162. 
Pflichten nichts geändert habe. ${ }^{468}$ Nichtsdestoweniger ebnete der durch die Rechtsprechung verursachte Modernisierungsimpuls den Weg für eine mögliche Aufwertung der Fürsorge und ihrer Leistungsempfänger, die von Fürsorgepolitikern gefordert wurde, ohne daß alle den Rechtsanspruch wirklich gewollt oder gar propagiert hätten. Nach diesem Urteil - so auch die Ansicht des Fachausschusses I - führte jedenfalls kaum ein Weg mehr an der expliziten Festlegung eines Rechtsanspruchs auf öffentliche Fürsorge im geplanten Bundesfürsorgegesetz vorbei. ${ }^{469}$

\section{Die Einfübrung des Warenkorb-Modells:}

die Verwaltungsvorschriften über den Aufbau der Richtsätze von 1955

Ein weiterer zentraler Modernisierungsschritt in der öffentlichen Fürsorge war 1955 die Etablierung des sogenannten Warenkorbs zur Ermittlung des laufenden notwendigen Lebensunterhalts. Die darin manifeste „Absicherung und Empirisierung des Bedarfsprinzips “ 470 war gleichzeitig Ausdruck wie Katalysator eines sich wandelnden Armutsbegriffs und stand in engem Zusammenhang mit der Entlastung der Fürsorge durch andere sozialpolitische Instrumentarien, mit der zunehmenden wirtschaftlichen Prosperität und nicht zuletzt den gerichtlichen Konsequenzen des Sozialstaatsgebots des Grundgesetzes. Bemerkenswerterweise waren es gerade die sozialpolitisch konservativen kommunalen Spitzenverbände, die im Zusammenspiel mit DV und Bundesinnenministerium das Warenkorb-Modell installierten, um auf diese Weise einer weiteren „Politisierung“ und „Parlamentarisierung“ der Richtsatzfrage begegnen zu können. ${ }^{471}$ Dadurch wurde letztlich eine erste klar bedarfsorientierte „Sockelung“ der Arbeiter- und Armenpolitik von der Fürsorge her ${ }^{472}$ in die Wege geleitet.

Formal galt bislang noch immer der Richtsatzerlaß von 1941, der u.a. bestimmte, daß die Richtsätze den notwendigen Bedarf an Nahrung, Beleuchtung, Kochfeuerung, Instandhaltung von Kleidung, Wäsche und Schuhwerk, Reinigung und „kleinere Bedürfnisse“, nicht aber den für Unterkunft abdecken sollten. ${ }^{473}$ Neben abgestuften Richtsatztypen (Haushaltsvorstand, Haushaltsangehörige unter bzw. über 16 Jahre, Alleinstehende) hatte der Erlaß auch ein Unterstützungsmaximum in Form der sogenannten Auffanggrenze definiert, das allerdings von

$468 \mathrm{Vgl}$. Knoll, Auswirkungen.

469 Vgl. Niederschrift über die Sitzung des Fachausschusses I am 8.10.1954, BAK, B 172444-01/5.

470 Leibfried u.a., Ende, S. 126.

471 An dieser Stelle kann sich die Darstellung auf einen Überblick zu diesem wichtigen Thema beschränken, denn die zunehmende „Bedarfsorientierung“ der Richtsatzpolitik bis hin zur Etablierung des Warenkorb-Modells bildet das Kernthema von Heisigs Untersuchung und wird dort - vor allem unter dem Blickwinkel der Aktivitäten der kommunalen Spitzenverbände - ausführlich behandelt; vgl. Heisig, Armenpolitik, 1995, bes. S. 101ff. Knapper, aber auch systematischer: Leibfried u.a., Ende; dies., Sozialpolitik.

472 Leibfried u.a., Sozialpolitik, S. 49.

473 Vgl. RMBliV. 1941, S. 1951; die „kleineren Bedürfnisse“ bezogen in der Regel nicht kulturellen Bedarf mit ein; vgl. Oel, Richtsätze, S.324. 
vielen Ländern mittlerweile abgeändert worden war. ${ }^{474}$ Festzusetzen waren die Richtsätze von den obersten Landesbehörden (also den Sozial- bzw. Innenministerien) oder den von ihnen beauftragten Stellen, d.h. den Verwaltungsorganen der Bezirksfürsorgeverbände. Obwohl die Richtsätze eigentlich keine Unterstützungssätze, sondern nur eine örtliche Meßlatte für den laufenden durchschnittlichen Lebensunterhalt sein sollten, fungierten sie in der Praxis als schematisch angewendete Festsätze und diktierten damit den Lebensstandard der Fürsorgeempfänger. ${ }^{475}$

„Daß die Fürsorgerichtsätze dem Preisgefüge nicht entsprechen und damit [...] nicht ausreichen, den primitiven Lebensunterhalt zu garantieren", war Anfang der fünfziger Jahre selbst innerhalb des DLT gängige Münze. ${ }^{476}$ Die bisherige Erfahrung hatte gelehrt, daß vor allem vor Landtagswahlen die zuständigen Länderministerien dem Druck aus den Parlamenten nachgaben und die (Rahmen-) Richtsätze erhöhten oder in der Vorweihnachtszeit Teuerungszulagen empfahlen und damit andere Länder unter Zugzwang brachten. ${ }^{477}$ Der parlamentarische Druck rührte nicht zuletzt aus der Tatsache, daß Anfang der fünfziger Jahre unter den Fürsorgeempfängern die Rentenbezieher noch immer die stärkste Gruppe bildeten, denen als umworbene Wählergruppe immer wieder eine gewisse Anpassung der Richtsätze an die steigenden Lebenshaltungskosten zugestanden wurde. ${ }^{478}$

Im Zuge der allgemeinen Diskussionen über die „Sozialreform“ wurde in den Landtagen die Forderung erhoben, die Richtsätze an Erhöhungen der Versicherungs- und Versorgungsleistungen zu koppeln. ${ }^{479}$ Ein solches Pendant zu den gefürchteten Rentenfreilassungen oder deren automatische Anpassung an die Entwicklung des Lebenshaltungskostenindexes ${ }^{480}$ oder gar des Bruttosozialprodukts oder die allgemeine Lohnentwicklung hätte die Richtsätze aus der fiskalpoliti-

${ }^{474}$ Laut Richtsatzerlaß sollte die Unterstützung im allgemeinen nicht höher sein als $85 \%$ des Netto-Arbeitseinkommens, das der Hilfsbedürftige bisher verdienen konnte; fehlte diese Vergleichsmöglichkeit, sollten $85 \%$ des Normaleinkommens der Bevölkerungsschicht zugrundegelegt werden, zu der der Unterstützte bislang gehört hatte. Geldknappheit, Massennot und die fehlenden Nachweismöglichkeiten bei Flüchtlingen hatten viele Länder dazu veranlaßt, statt dieser individuellen und großzügigen nun eine generelle Auffanggrenze festzulegen (z.B. 85\% des durchschnittlichen Einkommens eines ungelernten Hilfsarbeiters im örtlich vorherrschenden Gewerbezweig), die allerdings vor allem bei Familien mit mehreren Kindern zu erheblichen Härten geführt hatte; vgl. Oel, Auffanggrenze.

475 Vgl. Hans Muthesius, Vorbemerkungen, in: Öffentliche Einkommenshilfe, S. 1f.; ferner Auerbach, Einkommenshilfen, S.220; Oel, Richtsätze, S. 324.

476 Die Selbstverwaltung 4 (1950), S.165. Entsprechend die Einschätzung der Sozialminister der Westzonen auf ihrer ersten Konferenz am 22./23.2.1949 in Königstein/Taunus; vgl. NDV 29 (1949), S. 67.

477 Bereits die Preissteigerungen nach der Währungsreform hatten 1949 sämtliche Länder mit Ausnahme von Bayern zu einer Erhöhung der Richtsätze oder Gewährung von Teuerungszulagen veranlaßt; vgl. Heisig, Armenpolitik, 1995, S. 75ff., $111 \mathrm{f}$.

478 Vgl. Leibfried u.a., Ende, S. 127f.; auch Hockerts, Entscheidungen, S. 193.

479 Vgl. etwa für Nordrhein-Westfalen 1954 Heisig, Armenpolitik, 1995, S.118f.; ferner Leibfried u.a., Ende, S. 128.

480 Vgl. NDV 33 (1953), S. $74 f f$. 
schen Umklammerung gelöst, den Städten und Gemeinden damit weiteren sozialund finanzpolitischen Spielraum genommen und stand zudem in deutlichem Gegensatz zum restriktiven Hilfsbedürftigkeits-Begriff vieler Fürsorgepolitiker vor allem in den Landkreisen. ${ }^{481}$

Auch im Bundestag hatten vor allem SPD und KPD seit 1950 immer wieder wenn auch lange erfolglos - Initiativen des Bundes zur Erhöhung der Richtsätze gefordert. ${ }^{482}$ Erst das Fürsorgeänderungsgesetz bot dazu einen geeigneten Hebel: Sollte das Mehrbedarfs-Konzept bundesweit praktische Wirkung zeigen, war dessen Konkretisierung durch vertretbare Richtsätze erforderlich, zumal das neue Gesetz entsprechende neue Rahmenvorschriften vorsah und die regional oft sehr unterschiedlichen Richtsätze kaum mehr plausibel zu machen waren. ${ }^{483}$ Bereits seit Herbst 1951 hatten die kommunalen Spitzenverbände versucht, stärker auf die unausweichliche zentrale Richtsatzsteuerung Einfluß zu nehmen. ${ }^{484}$ Ein gesetzlich garantiertes Anhörungsrecht bei der Ausarbeitung der Verwaltungsvorschriften konnten sie zwar nicht durchsetzen, doch ihr konkreter Einfluß blieb gewahrt, zumal Gottschick schon im März 1952 bemerkt hatte, daß von seinem Haus „eine derartige Beteiligung [...] bereits ohne ausdrückliche Vorschrift gewollt" sei. ${ }^{485}$

Um zu verhindern, daß Städte und Landkreise von Parlamentsbeschlüssen oder verwaltungsgerichtlichen Überprüfungen der Richtsätze schließlich überrollt würden, plädierte Ende 1953 etwa Theodor Marx vom DST für eine „hinreichende wissenschaftliche Bearbeitung“ der Richtsatzfrage, die er wie die DLT-Führung im hierfür prädestinierten DV angesiedelt wissen wollte. ${ }^{486}$ Eine solche „Verwissenschaftlichung“ der Richtsatzbemessung korrelierte mit der sozialreformerischen Stoßrichtung einer stärkeren Bedarfsorientierung, verlangte diese doch nach einer möglichst nicht anfechtbaren allgemeinen Definition eben dieses Bedarfs. Trotzdem war diese Linie innerhalb der kommunalen Spitzenverbände keineswegs unumstritten: Der von den „Modernisten“ (Heisig) in ihren Reihen betriebenen Suche nach neuen, politisch unangreifbaren allgemeinen Unterstützungsmaßstäben widersetzten sich die besonders in den bayerischen Kommunalverbänden beheimateten „Traditionalisten“, die nach wie vor einer möglichst

481 Vgl. Heisig, Armenpolitik, 1995, S. 104f. Der Hessische Städtetag jedoch war mit gewissen Richtsatzanhebungen einverstanden, u.a., ,um den Hilfsbedürftigen eine vertretbare Beteiligung an der Steigerung des Sozialproduktes nicht vorzuenthalten“; Schreiben des Hessischen Städtetages an den Hessischen Minister des Innern vom 10.9.1954, Abschrift, LAB, B Rep. 142-9, 1332.

482 Vgl. Antrag der SPD vom 29.7.1950, BT, 1. Wp. 1949, Anlagen, Bd. 5, Drs. 1271; Antrag der KPD vom 13.10.1950, ebenda, Bd.7, Drs. 1471; Interpellation der SPD vom 13.2. 1951, ebenda, Bd.9, Drs. 1937; Antrag der KPD vom 21.3.1951, ebenda, Bd.10, Drs. 2087.

483 So auch Kitz, Gegenwartsfragen, S. 333, auf der Sozialministerkonferenz im Juli 1954 in Stuttgart.

${ }^{484} \mathrm{Vgl}$. Heisig, Armenpolitik, 1995, S. 85ff.

485 Gottschick auf der Sitzung des gemischten Unterausschusses des DST und des DLT am 5.3.1952, Niederschrift, LAB, B Rep. 142-9, 1331.

486 Vgl. Marx an Hans Dellbrügge am 7.12.1953, LAB, B Rep. 142-9, 1332; ferner Heisig, Armenpolitik, 1995, S. 88ff. 
weitgehenden Individualisierung das Wort redeten. 487 Tatsächlich avisierte der Fachausschuß I Anfang Februar 1954 zunächst noch ohne praktische Konsequenzen die Bildung eines Arbeitskreises „Aufbau der Richtsätze“.488

Wenn das Innenministerium die Ausarbeitung der Richtsatzrichtlinien aber nach wie vor eher dilatorisch behandelte, lag das vermutlich an der geplanten Umstellung der Kriegsfolgenhilfe-Abrechnung. Da die künftigen Pauschalleistungen des Bundes auf der Grundlage der tatsächlichen Ausgaben 1953/54 errechnet werden sollten, war der Bundesfinanzminister an möglichst niedrigen Richtsätzen interessiert - eine forcierte Angleichung der Richtsätze auf höherem Niveau durch das Innenministerium konnte also ruhig noch eine Weile warten.

Im Juni 1954 forderte Walter Auerbach auf der Sozialministerkonferenz in Stuttgart eine möglichst angeglichene Richtsatzgestaltung auf der Grundlage einheitlicher Bemessungsfaktoren ${ }^{489}$, und seit dem Frühherbst 1954 wurden in zahlreichen Ländern auf Druck der Landtage abermals die Richtsätze erhöht. 490 Auch im Bundestag beantragte die SPD Anfang September zusammen mit dem Entwurf für ein neues Rentenzulagengesetz, die Bundesregierung solle mit den Ländern Richtsatzerhöhungen aushandeln. ${ }^{491}$ Nachdem der Bundestag den Antrag Ende September an den Fürsorgeausschuß überwiesen hatte ${ }^{492}$, trat der Arbeitskreis „Aufbau der Richtsätze“ des DV endlich am 7. Oktober im Auftrag des Bundesinnenministeriums zusammen und leistete in nur wenigen Monaten die für die bundesweite Etablierung des Warenkorb-Prinzips entscheidenden Vorarbeiten. Die Zusammensetzung des Arbeitskreises bot die Gewähr, daß die unterschiedlichen Interessen der verschiedenen Kostenträger der Fürsorge zur Geltung kamen, Vertreter der Fürsorgeempfänger hingegen wurden weder jetzt noch später bei der Ausarbeitung der Verwaltungsvorschriften hinzugezogen, wenn auch das Bundesministerium für Vertriebene auf der ersten Sitzung vertreten war. ${ }^{493}$ Aufgabe des Arbeitskreises war nicht die Definition eines Richtsatzes in verbindlichem DM-Betrag; vielmehr sollte ein Bedarfsschema für den Aufbau der Richtsätze und eine wissenschaftlich abgesicherte Abstufung nach Altersgruppen entwickelt werden. In der Praxis führte der Weg dann aber gerade über eine in DM-Preisen ausgedrückte Bestimmung des Existenzminimums

487 Vgl. Heisig, Armenpolitik, 1995, S. $120 f$.

488 Vgl. Niederschrift über die Sitzung des Fachausschusses I am 4.2.1954, LAB, B Rep. 142-9, 1256.

489 Vgl. Auerbach, Standort, S. 335.

490 Vgl. Heisig, Armenpolitik, 1995, S. 111ff.

491 Vgl. SPD-Antrag vom 1.9.1954, BT, 2. Wp. 1953, Anlagen, Bd. 31, Drs. 789.

492 Vgl. Bundestagssitzung am 24. 9. 1954, BT, 2. Wp. 1953, Sten. Ber., Bd. 21, S. 2214.

493 Dem von Muthesius geleiteten Arbeitskreis gehörten u.a. an: Vertreter/innen der Sozialministerien von Niedersachsen, Nordrhein-Westfalen, Schleswig-Holstein, Hamburg und Baden-Württemberg, für den DST Hildegard Schräder, Stadtdirektor Ernst Weinbrenner (Duisburg, CDU) und Heinz Keese (Hannover, SPD), für den DLT Johann Bangert und der Buchener Landrat Franz-Xaver Schmerbeck (CDU), sowie für den DV u.a. Geschäftsführer Pense und Referent Krug von Nidda. Gast war u.a. Gottschick. Neben Sachverständigen für Statistik zog der Ausschuß vor allem den Ernährungsphysiologen Heinrich Kraut hinzu; vgl. Teilnehmerliste der Sitzung des Arbeitskreises „Aufbau der Richtsätze“ am 7. 10.1954, LAB, B Rep. 142-9, 1264. 
und damit seine automatische Bindung an die Entwicklung der Lebenshaltungskosten. 494

Während der DV in den Jahren der Weimarer Republik eine wissenschaftliche Bestimmung des Existenzminimums abgelehnt hatte, griff der Arbeitskreis jetzt auf Vorarbeiten aus dieser Zeit, insbesondere das damals im Kölner Wohlfahrtsamt unter Hertha Kraus entwickelte Warenkorb-Prinzip zurück. ${ }^{495}$ Anhand ernährungswissenschaftlicher Vorgaben, ergänzt durch Erfahrungen aus der Fürsorgepraxis wurde ein theoretischer Warenkorb aus Nahrungsmitteln und Dienstleistungen zusammengestellt und mit Ergebnissen einer hessischen Verbrauchsstatistik über die Kosten des Lebensbedarfs unterer Einkommensgruppen verglichen, um den berechneten Bedarf durch die tatsächlichen Verbrauchsgewohnheiten zu überprüfen. In Preisen ausgedrückt sollte der so ermittelte Warenkorb dann die Grundlage für wissenschaftlich fundierte, stabilere und leichter an die Entwicklung der Lebenshaltungskosten anzupassende Richtsätze bilden. ${ }^{496}$

Die möglichen Ergebnisse würden weit über die Fürsorge hinaus Bedeutung erlangen; schließlich, so der Arbeitskreisvorsitzende Muthesius, hielten es „die Gesetzgeber für ihre selbstverständliche Pflicht [...], andere soziale Einkommenshilfen unter allen Umständen so zu konstruieren, daß der durch sie garantierte Lebensstandard merklich höher liegt, als der durch die Richtsätze der öffentlichen Fürsorge vermittelte“. ${ }^{497}$ Und so stieß die Tätigkeit des Arbeitskreises nicht nur auf das Interesse des Bundesfinanzministeriums, dessen extensives Bedarfskonzept nach einem abgesicherten Maßstab verlangte; auch im „Beirat“ des Arbeitsministeriums erhoffte man sich Material für die Bemessung von möglichen Mindestsozialleistungen bis hin zur konkreten Formulierung der Rentenformel. ${ }^{498}$

Während der Arbeiten am Warenkorb hatte auch der Fürsorgeausschuß des Bundestages den seinerzeitigen SPD-Antrag behandelt und - in Anerkennung der politischen und rechtlichen Realitäten - auf die Frage der Verwaltungsvorschriften umgelenkt. Der am 17. Dezember 1954 vom Bundestag einstimmig angenommene Ausschußantrag forderte die Bundesregierung dazu auf, bei ihren „Bemü-

${ }^{494}$ Zur Vorgehensweise des Arbeitskreises vgl. Kraut, Sicherung, S. 6ff.; ferner Heisig, Armenpolitik, 1995, S.114ff.

$495 \mathrm{Zu}$ den verschiedenen Bemühungen bis 1945 um die Ermittlung des wirtschaftlichen Existenzminimums anhand von Haushaltsbüchern, Einkommens- und Verbrauchsstatistiken, ernährungsphysiologischen Untersuchungen etc. vgl. Heisig, Armenpolitik, 1995, S. 79ff., 113f.; ders., Armenpolitik, 1990, S. 556f., 589f. Ende der vierziger Jahre hatte die ehemalige Kraus-Mitarbeiterin Hildegard Schräder auf der Grundlage des international anerkannten Ernährungsbedarfs von 2400 Kalorien für Kölner Verhältnisse einen Nahrungsmittel-Warenkorb zusammengestellt, diesen um den im Richtsatzerlaß von 1941 vorgesehenen zusätzlichen laufenden Bedarf für Beleuchtung etc. ergänzt, in aktuelle Preise umgerechnet und danach einen Richtsatz für einen Haushaltsvorstand oder Alleinstehenden von 50,00 DM ermittelt.

496 Zu den Rückwirkungen der Erhebungsgrundsätze der Preisstatistik auf die Zusammensetzung des Warenkorbs vgl. Galperin, Sozialhilfe, S. 156ff.

497 Muthesius, Vorbemerkungen, in: Öffentliche Einkommenshilfe, S. 1.

498 Vgl. Auerbach, Standort, S. 335; ders., Einkommenshilfen, S.220; ders.: Mut zur sozialen Sicherheit, in: ders., Beiträge, S. 57; Oel, Richtsätze, S. 323; Keese, Betrachtungen, S. 114; Marx, Die Städte und die Neuordnung, S.33f. 
hungen um einen den Zeitverhältnissen angepaßten Aufbau der Richtsätze [...] sicherzustellen, daß die Sicherung des notwendigen laufenden Lebensbedarfs gewährleistet wird“.499 Dabei sollten auch kulturelle Bedürfnisse berücksichtigt werden. Die von vielen Landkreisen favorisierte Beibehaltung von Ortsklassen (niedrigere Richtsätze in kleinen Gemeinden) hielt der Ausschuß für nicht mehr zeitgemäß. ${ }^{500}$

Ende Januar 1955 schloß der Arbeitskreis seine Arbeit am Warenkorb ab501: Er plädierte dafür, in den Richtsatz wie bisher nur die Kosten für Nahrung, Kochfeuerung und Beleuchtung, Instandhaltung von Kleidung etc., Reinigung und Körperpflege einzubeziehen, darüber hinaus jetzt auch „andere Ausgaben“ (kleine Ergänzungen des Hausrats, Briefmarken, Streichhölzer, Straßenbahnkarte, Zeitung u.ä.). ${ }^{502}$ Dazu legte der Arbeitskreis umfangreiche, nach Alter, teilweise Geschlecht und Haushaltsstand differenzierende Tabellen über die jeweils für notwendig erachteten monatlichen Verbrauchsgüter und -mengen („Warenkorb“) sowie die dafür veranschlagten Ausgaben vor. Dabei handelte es sich um einen Minimalbedarf, berechnet für nicht arbeitende Personen, wobei allerdings „die Ernährung der Fürsorgeempfänger zwar in der Auswahl der Lebensmittel so sparsam wie möglich, aber doch zur Deckung ihres Bedarfs an Energieträgern und Aufbaustoffen voll ausreichend sein" sollte. ${ }^{503}$ Auf Betreiben Johann Bangerts vom DLT waren dabei allerdings bewußt niedrige Bundesdurchschnittspreise von 1954 angesetzt worden, so daß aus den Tabellen keine drastischen Richtsatzerhöhungen zu begründen waren. ${ }^{504}$ So veranschlagte der Arbeitskreis als monatlichen Gesamtaufwand für den Haushaltsvorstand bzw. Alleinstehenden (= Eckrichtsatz, ohne Verlustzuschlag) 55,61 DM. ${ }^{505}$ Der aktuelle Richtsatz im Bundesdurchschnitt betrug 52,10 DM..$^{506}$

Für die Abstufung der einzelnen Richtsatztypen gegenüber dem Eckrichtsatz $(=100 \%)$ schlug der Arbeitskreis höhere Relationen als bisher vor: für Haushalts-

499 Vgl. Mündlicher Bericht des Ausschusses für Fragen der öffentlichen Fürsorge vom 10.11.1954, BT, 2. Wp. 1953, Anlagen, Bd.32, Drs. 1008, sowie Bundestagssitzung vom 17.12.1954, ebenda, Sten. Ber., Bd.22, S. 3287.

500 Vgl. den Schriftlichen Bericht des Ausschusses für Fragen der öffentlichen Fürsorge vom 17.12.1954, BT, 2. Wp. 1953, Anlagen, Bd. 32, zu Drs. 1008.

501 Die im Arbeitskreis gehaltenen Referate wurden - teilweise geändert - mit den Abschlußempfehlungen im Herbst 1955 von Muthesius als DV-Schrift veröffentlicht; vgl. Öffentliche Einkommenshilfe; dazu Oel, Richtsätze; Auerbach, Einkommenshilfen; NDV 36 (1956), S. $9 \mathrm{ff}$.

502 Vgl. „Zusammenfassung der Ergebnisse des Arbeitskreises ,Aufbau der Richtsätze““, in: Öffentliche Einkommenshilfe, S.129-133, nebst Tabellen. Wie bisher sollte neben der Miete auch die Winterfeuerung und Anschaffung von Kleidung, Wäsche etc. nicht einbezogen werden, da sonst eine zu starke Erhöhung der Richtsätze und damit ein Ansteigen der Zahl der zu Unterstützenden befürchtet wurde, vgl. ebenda, S. 130. Gegen eine solche Einbeziehung wandte sich vor allem der Vorsitzende des DLT-Sozialausschusses Schmerbeck, Bezirksfürsorgeverbände, S. 322.

${ }^{503}$ Heinrich Kraut: Die Sicherung des Nahrungsbedarfes, in: Öffentliche Einkommenshilfe (1955), S. 4-19, hier S. 5.

504 Vgl. Heisig, Armenpolitik, 1995, S. 117.

505 Vgl. Tabelle 4, in: Öffentliche Einkommenshilfen, S. 59.

506 Vgl. Oel, Richtsätze, S. 326. 
angehörige über 6 Jahren $80 \%$, Kinder unter 6 Jahren $60 \%$, sowie als Sonderregelung für Jugendliche zwischen 14 und 18 Jahren 100\%, da die bisherigen Richtsätze deren besonderen Nahrungsbedarf kaum berücksichtigt gelassen hätten.507 Über diese Ergebnisse hinaus befürwortete der Vorstand des DV für Alleinstehende einen individuellen Zuschlag zwischen 5 und $15 \%$, da sie eventuelle Bedarfsschwankungen schwieriger ausgleichen könnten als eine Haushaltsgemeinschaft. ${ }^{508}$ Außerdem plädierte der Vorstand für eine Erhöhung der veranschlagten Ausgaben für Nahrungsmittel um 5 bis $10 \%$, um Verluste durch unrationellen Einkauf oder Verderb aufzufangen. Zwar lehnten auch viele Arbeitskreismitglieder eine Staffelung nach Ortsklassen $a b^{509}$, doch die offizielle Zusammenfassung der Arbeitsergebnisse machte zu diesem Punkt keine konkreten Vorgaben.

Bei der brisanten Frage der „Auffanggrenze“ blieb der Arbeitskreis eher unbestimmt. Bislang hatte diese Grenze vor allem hilfsbedürftige Familien mit mehreren Kindern getroffen, da ihre kumulierten Richtsätze noch am ehesten zum Vergleich herangezogene Niedriglöhne überschritten. Die Auffanggrenze, die den Anreiz zur Arbeit erhalten sollte und auch dem Gerechtigkeitsempfinden des Steuerzahlers geschuldet war, benachteiligte also vor allem diejenigen, deren Förderung ein erklärtes Anliegen der Fürsorge war. Mit Einführung des Kindergeldes ab 1.Januar 1955 würde die Auffanggrenze allerdings so nach oben verschoben, daß sie allenfalls für Familien mit 5-7 Kindern noch eine Rolle spielte. ${ }^{510}$ Der Arbeitskreis empfahl daher, in den Verwaltungsvorschriften keine generelle Grenze mehr vorzusehen, sondern eine Regelung, „die im Einzelfall für die Unterstützten Härten vermeidet“ ${ }^{511}$ Inwieweit dieser Kompromiß überhaupt noch mit dem seit einem halben Jahr verbrieften Rechtsanspruch auf Gewährung des notwendigen Lebensunterhalts vereinbar war, blieb offen.

Die im Herbst 1955 veröffentlichten Empfehlungen des Arbeitskreises bildeten die Grundlage für den im Bundesinnenministerium ausgearbeiteten Entwurf für die „Verwaltungsvorschriften über den Aufbau der Fürsorgerichtsätze und ihr Verhältnis zum Arbeitseinkommen". 512 Wie die Vorschriften nochmals betonten, waren die Richtsätze „keine festen Unterstützungssätze“, sondern dienten deren Berechnung „im Regelfall“ (Ziff. 1). Bei den einzelnen Bedarfsgruppen folgte das Innenministerium den Vorschlägen des Arbeitskreises, wobei es bei dem Nahrungsbedarf noch einen Aufschlag von 5\% für den Verlust einberechnete und damit einen Eckrichtsatz von 57,32 DM ermittelte. Anders bei dem Verhältnis der

507 Vgl. Kraut, Sicherung des Nahrungsbedarfes, in: Öffentliche Einkommenshilfe, S. 11. Der Richtsatzerlaß von 1941 sah für Haushaltsangehörige unter 16 Jahren 40-50\%, für über 16jährige 70-80\% des Eckrichtsatzes vor.

508 Bisher hatte der Richtsatz für Alleinstehende automatisch 110\% des Eckrichtsatzes betragen.

509 Vgl. Öffentliche Einkommenshilfe, S. 44f., 74.

510 Dies war vom DLT zunächst heftig bestritten worden, so daß eine Umfrage in ausgewählten Stadt- und Landkreisen mit dem genannten Ergebnis veranstaltet wurde; vgl. Heisig, Armenpolitik, 1995, S. 116 f.

511 Zusammenfassung, in: Öffentliche Einkommenshilfe, S. 133.

512 BR 1955, Drucksachen, Drs. 395/55. Heisig, Armenpolitik, 1995, S. 125, behandelt die Vorarbeiten im BMI sowie die Verwaltungsvorschriften nur noch knapp. 
Richtsätze für die verschiedenen Personengruppen: Die Richtsätze für Kinder und Jugendliche blieben hinter den Vorschlägen des Arbeitskreises zurück und ließen Spannbreiten $\mathrm{zu}^{513}$; obwohl vom Ministerium selbst eingeholte zusätzliche Gutachten abermals den erhöhten Bedarf gerade der Jugendlichen bestätigt hatten ${ }^{514}$, fürchtete man in der Sozialabteilung die Auswirkungen höherer Richtsätze auf Niedriglöhne und Renten. ${ }^{515}$ Für alleinlebende Alleinstehende sah der Entwurf einen Zuschlag von „in der Regel 10 v.H.“ vor (Ziff. 3) und bewegte sich damit innerhalb der DV-Vorgaben. Wie bisher wurde der Aufwand für die Unterkunft nicht in den Richtsatz einbezogen, sondern sollte in der Regel in Höhe der tatsächlichen Kosten ausgezahlt werden. Die viel diskutierte "Auffanggrenze“ entfiel praktisch ersatzlos: Zwar forderte der Entwurf prinzipiell, daß die Richtsätze zuzüglich durchschnittlicher Mietbeihilfen insgesamt unter dem regional errechneten durchschnittlichen Netto-Einkommen unterer Lohngruppen zuzüglich Kindergeld bleiben sollten, doch im Einzelfall müsse die Unterstützung den notwendigen Lebensunterhalt sichern. ${ }^{516}$

Zusammen mit dem Entwurf übersandte die Bundesregierung am 3. Dezember 1955 offiziell auch die vom Arbeitskreis erstellten Warenkorb-Tabellen. Durch deren Veröffentlichung als Bundesrats-Drucksache verfolgte man von seiten des Innenministeriums eine doppelte Strategie: Einerseits wurden die Landesregierungen damit indirekt an die Erfüllung zumindest der im Warenkorb festgeschriebenen Minimalbedarfe gebunden ${ }^{517}$ und damit die behördlichen Ermessensräume stärker und dauerhaft eingeschränkt. Andererseits wollte man den Länderministern wissenschaftliches Material gegen Forderungen nach Richtsatzerhöhungen, die nicht durch gestiegene Lebenshaltungskosten zu begründen waren, an die Hand geben. ${ }^{518}$

513 Vor allem die Sonderregelung für heranwachsende Haushaltsangehörige (Richtsatz von $100 \%$ ) hatte der Entwurf nicht übernommen, sondern sah in Ziff. 3 einen Richtsatz von 80-90\% des Eckrichtsatzes vor; dieser sollte dann aber auch für die 18-20jährigen gelten (Ziff. 2). Auch weiterhin sollten bei den übrigen Richtsatzgruppen für Kinder und Jugendliche größere Spielräume bestehen bleiben (Kinder bis 6 Jahre 50-60\%, 7-13jährige 70-80\%); erfahrungsgemäß hatten die Länder aber bisher immer die Untergrenze gewählt. Besonders umstritten war die Begrenzung der Richtsätze für Auszubildende auf $120 \%$ des Eckrichtsatzes, was den Mehrbedarfsvorschriften des FÄG eigentlich widersprach und deshalb von verschiedenen Ländern auch nicht übernommen wurde; vgl. Keese, Betrachtungen; Auerbach, Fürsorgerichtsätze, S. 40f.

514 Vgl. Öffentliche Einkommenshilfe, S. 20ff.

515 Vgl. Kitz auf der Sitzung des DST-Sozialausschusses am 5./6. 5. 1955, LAB, B Rep. 1429, 1332. Ähnliche Befürchtungen hegten auch einige Länder: Muthesius berichtete 1956, kurz vor Veröffentlichung der Arbeitskreis-Ergebnisse sei er „von einigen Länderarbeitsministern dringend gebeten worden, das nicht $\mathrm{zu}$ tun, weil sie fürchteten, dass durch diese Veröffentlichungen ein grosser Teil ihrer Tarifverträge mit geringen Löhnen in Gefahr geraten würde!“, Vortrag auf der Sitzung des DLT-Sozialausschusses am 5.9. 1956, Ms., BAK, B 106-9697.

516 Der Vergleich mit dem Arbeitseinkommen war jetzt also nur noch bei der Festsetzung der Richtsätze anzustellen, nicht aber mehr bei deren Anwendung; vgl. Gottschick, Verwaltungsvorschriften, S. 38 .

517 Vgl. Begründung zum Entwurf der Verwaltungsvorschriften, S. 3; BR, Drucksachen 1955, Bd. 5, Drs. 395/55.

518 Vgl. Heisig, Armenpolitik, 1995, S. 133, Anm. 155. 
Doch bereits zuvor waren Entwurf und Tabellen durch einen Korrespondenten der United Press publik gemacht worden. Sofort hagelte es öffentlich Kritik an den - so der Tenor - viel zu niedrig angesetzten Richtsätzen des Arbeitskreises, die das Bundesinnenministerium als Berechnungsgrundlage weiterzuempfehlen gedachte. ${ }^{519}$ Der Bundesrat vereinfachte den Entwurf dann insofern, als er sämtlichen Haushaltsangehörigen ab 14 Jahren einen Richtsatz von 80-90\% des Eckrichtsatzes zugestand und stimmte ihm im übrigen am 21. Dezember zu, so daß die Verwaltungsvorschriften unter dem 23. Dezember 1955 erlassen und mit einer sechsmonatigen Übergangsfrist in Kraft treten konnten. ${ }^{520}$ Die Fachöffentlichkeit hingegen begrüßte prinzipiell den Mut des DV und des BMI, erstmals eine Definition des notwendigen Lebensbedarfs überhaupt zu wagen, so daß sich die Fürsorgeleistungen zum ersten Mal ,jenem sozialen Existenzminimum“ näherten, „auf das jeder ordentliche Bürger in einem sozialen Rechtsstaat einen Anspruch hat". .521

Tatsächlich führten die Untersuchungen des Arbeitskreises und die Verwaltungsvorschriften allerorten „zu einer bemerkenswerten Erhöhung“522 und stärkeren Angleichung der Richtsätze und Richtsatzklassen - ein Eingeständnis, daß die bisherigen Sätze den notwendigen Lebensbedarf gerade der Kinder und Jugendlichen kaum gedeckt hatten. ${ }^{523}$ Deren Neuregelung, vor allem die erlaubten Spannbreiten, war in den Augen vieler Fachleute allerdings wenig befriedigend: $\mathrm{Da}$ tatsächlich bald fast alle Länder die Untergrenze der neuen Prozentverhältnisse wählten, waren die Jugendlichen auch zwei Jahre später noch „unter allen Gruppen der Fürsorgeberechtigten am weitesten von einer Deckung des Bedarfes für den notwendigen Lebensunterhalt entfernt “524.

Der maßgeblich an der Ausarbeitung des Warenkorbs von 1955 beteiligte Ernährungswissenschaftler Heinrich Kraut räumte wenige Jahre später ein, daß dieser „auf die billigste Weise kalkuliert“ ${ }^{\text {“25 }}$ gewesen sei, und stellte damit offen

519 Vgl. ebenda, S. 118; ferner entsprechende Berichte etwa im Berliner Tagesspiegel vom 3.2.1956 und im Wuppertaler Generalanzeiger vom 2.2.1956.

520 GMBl. 1956, S. 58; vgl. NDV 36 (1956), S. 54ff.

521 So der selbst im Arbeitskreis tätig gewesene Hannoveraner Sozialamtsleiter Heinz Keese, Betrachtungen, S. 115. Ähnlich Oel, Neuordnung der Fürsorgerichtsätze, S. 213f.; kritisch Manderschied, Neuordnung; Auerbach, Fürsorgerichtsätze; vgl. auch SF 5 (1956), S. $92 f$.

522 Keese, Betrachtungen, S. 114; dieser schätzte den finanziellen Mehraufwand nur für Niedersachsen auf fast 10 Mio. DM jährlich. Zu den Richtsatzerhöhungen vgl. die Übersicht bei Oel, Neuordnung der Fürsorgerichtsätze, S. 215. Der Eckrichtsatz wurde in den Ländern gegenüber November 1955 bis April 1956 um 2-20\% erhöht und betrug dann zwischen 52 DM (Mindestrichtsatz in Bayern) und 72 DM (baden-württembergische Städte über 500000 Einwohner). Die Richtsätze für Jugendliche zwischen 14 und 16 Jahren wurden am stärksten angehoben; sie lagen im November 1955 zwischen 27 DM (Bayern, Mindestrichtsätze) und 38 DM (Berlin, Nordrhein-Westfalen, Höchstsatz) und wurden bis April 1956 in fast allen Ländern um rund 50\% erhöht.

523 Vgl. Oel, Neuordnung der Fürsorgerichtsätze, S. 216. Im Bundesdurchschnitt lag der vom BMI berechnete Minimalbedarf bei Kindern und Jugendlichen ab 7 Jahren um mindestens ein Drittel höher als die bisherigen Richtsätze (Bundesdurchschnitt); vgl. einen entsprechenden Vergleich bei Oel, Richtsätze, S. 326.

524 Kraut, Sicherung, S. 9. Vgl. ferner Auerbach, Fürsorgerichtsätze, S. $39 f$.

525 Kraut auf der Sitzung des Arbeitskreises „Aufbau der Richtsätze“ am 30.11.1960, LAB, B Rep. 142-9, 1264. 
dessen wissenschaftliche Fundierung in Frage. Das Zugeständnis der Preismanipulation sei allerdings die Voraussetzung dafür gewesen, das Warenkorbprinzip überhaupt durchzusetzen. ${ }^{526}$ Infolge der niedrigen Ausgangspreise und der erneuten Steigerung der Lebenshaltungskosten habe der Fürsorgeberechtigte schon 1956 nicht mehr mit den auf Preisen von 1954 basierenden Beträgen auskommen können. ${ }^{527}$ Erst die Überarbeitung des Warenkorbes 1961/62 brachte hier zunächst deutliche Erhöhungen.

Nichtsdestoweniger verbesserte die Etablierung des Warenkorbes mit seinen zentralen Merkmalen der „Stetigkeit, Einheitlichkeit und Dynamisierbarkeit" und der damit einhergehenden „Sprengung der Auffanggrenze“528 - ungeachtet der ihm nach wie vor zugrundeliegenden Werturteile ${ }^{529}$ und der problematischen nahezu legislatorischen Rolle des DV bei seinem Zustandekommen ${ }^{530}$ - seit 1955 die Lage der Unterstützungsempfänger nachhaltig: Der bislang vorherrschende Druck des (Niedrig-)Lohnniveaus auf die öffentliche Fürsorge (Prinzip der less eligibility) war weitgehend dem Prinzip der Bedarfsorientierung gewichen, das zumindest bis Anfang der 1980er Jahre Raum bot „für eine stetige, weitgehend entpolitisierte und [...] professionalisierte soziale Grundsicherung, also für ein historisch vergleichsweise respektables letztes Netz “531.

${ }^{526} \mathrm{Vgl}$. Kraut auf der Sitzung des Arbeitskreises am 9.2.1961, ebenda.

527 Vgl. Kraut, Sicherung, S. 9.

528 Leibfried u.a., Sozialpolitik, S. 48.

529 Die „verdeckten Wertungselemente“ bei der vermeintlich objektiven Warenkorbbestückung und ihre Konsequenzen für das Richt-/Regelsatzsystem bzw. das Sozialhilferecht erörtert Giese, Regelsatzsystem, S. 518ff. (hier S. 520).

530 Dazu Stolleis, Rechtsgrundlagen, S. 103; Oberbracht, Parlamentarisierung, S. 47ff., sowie Rudloff, Fürsorge, S. $198 \mathrm{f}$.

${ }^{531}$ Leibfried u.a., Ende, S. 126; vgl. auch Rudloff, Fürsorge, S. $197 f$. 